\title{
Mapping DNA interaction landscapes in psoriasis susceptibility loci highlights KLF4 as a target gene in $9 q 31$
}

Helen Ray-Jones ${ }^{1,2}$, Kate Duffus ${ }^{1}$, Amanda McGovern ${ }^{1}$, Paul Martin ${ }^{1,3}$, Chenfu Shi', Jenny Hankinson², Oliver Gough ${ }^{1}$, Annie Yarwood ${ }^{1,2}$, Andrew P. Morris ${ }^{1}$, Antony Adamson ${ }^{4}$, Christopher Taylor ${ }^{1}$, James Ding ${ }^{1}$, Vasanthi Priyadarshini Gaddi ${ }^{1}$, Yao Fu ${ }^{5}$, Patrick Gaffney ${ }^{5}$, Gisela Orozco ${ }^{1}$, Richard B. Warren ${ }^{2}$ and Steve Eyre ${ }^{1 *}$

\begin{abstract}
Background: Genome-wide association studies (GWAS) have uncovered many genetic risk loci for psoriasis, yet many remain uncharacterised in terms of the causal gene and their biological mechanism in disease. This is largely a result of the findings that over $90 \%$ of GWAS variants map outside of protein-coding DNA and instead are enriched in cell typeand stimulation-specific gene regulatory regions.

Results: Here, we use a disease-focused Capture Hi-C (CHi-C) experiment to link psoriasis-associated variants with their target genes in psoriasis-relevant cell lines (HaCaT keratinocytes and My-La CD8+ T cells). We confirm previously assigned genes, suggest novel candidates and provide evidence for complexity at psoriasis GWAS loci. For one locus, uniquely, we combine further epigenomic evidence to demonstrate how a psoriasis-associated region forms a functional interaction with the distant (> $500 \mathrm{~kb}$ ) KLF4 gene. This interaction occurs between the gene and active enhancers in HaCaT cells, but not in My-La cells. We go on to investigate this long-distance interaction further with Cas9 fusion protein-mediated chromatin modification (CRISPR activation) coupled with RNA-seq, demonstrating how activation of the psoriasisassociated enhancer upregulates KLF4 and its downstream targets, relevant to skin cells and apoptosis.
\end{abstract}

Conclusions: This approach utilises multiple functional genomic techniques to follow up GWAS-associated variants implicating relevant cell types and causal genes in each locus; these are vital next steps for the translation of genetic findings into clinical benefit.

Keywords: Psoriasis, Chromatin, GWAS, CHi-C, HiChIP, CRISPR, KLF4

\section{Background}

Psoriasis is a common immune-mediated disease that causes the formation of red, scaly plaques on the skin. On a cellular basis, psoriasis is thought to be driven by a complex interplay between the immune system, such as $\mathrm{T}$ cells and dendritic cells, and the resident cells of the

\footnotetext{
* Correspondence: steve.eyre@manchester.ac.uk

${ }^{1}$ Centre for Genetics and Genomics Versus Arthritis, Division of Musculoskeletal and Dermatological Sciences, School of Biological Sciences, Faculty of Biology, Medicine and Health, The University of Manchester, Manchester, UK

Full list of author information is available at the end of the article
}

epidermis. Keratinocytes are the most abundant cell type in the epidermis [1] and are highly dysregulated in psoriasis, undergoing accelerated differentiation and migration to the surface of the skin where they create the characteristic plaques [2]. T lymphocytes, such as CD4+ and $\mathrm{CD} 8+$, infiltrate the epidermis and release proinflammatory cytokines such as IL-17, IFN- $\gamma$ and IL-22 [3-5]. Keratinocytes respond to immune signals and release chemokines that can in turn recruit $\mathrm{T}$ cells and dendritic cells; this feedback loop leads to prolonged inflammation $[4,6]$.

C The Author(s). 2020 Open Access This article is licensed under a Creative Commons Attribution 4.0 International License, which permits use, sharing, adaptation, distribution and reproduction in any medium or format, as long as you give appropriate credit to the original author(s) and the source, provide a link to the Creative Commons licence, and indicate if changes were made. The images or other third party material in this article are included in the article's Creative Commons licence, unless indicated otherwise in a credit line to the material. If material is not included in the article's Creative Commons licence and your intended use is not permitted by statutory regulation or exceeds the permitted use, you will need to obtain permission directly from the copyright holder. To view a copy of this licence, visit http://creativecommons.org/licenses/by/4.0/. The Creative Commons Public Domain Dedication waiver (http://creativecommons.org/publicdomain/zero/1.0/) applies to the data made available in this article, unless otherwise stated in a credit line to the data. 
Genetic predisposition is the largest risk factor for psoriasis. Genome-wide association studies (GWAS) have so far identified 63 loci associated with susceptibility in individuals of European ancestry [7] and more than 20 further unique signals in the Han Chinese population $[8,9]$. The majority of single nucleotide polymorphisms (SNPs) associated with psoriasis and other immune-mediated conditions do not map within gene coding regions; rather, they are enriched in non-coding enhancer elements [10], with approximately $60 \%$ of predicted causal SNPs lying within cell type-specific immune enhancers relevant to the disease of interest and approximately $8 \%$ in promoters [11, 12]. Historically, gene candidates were assigned to GWAS loci based on proximity or biological function; however, this can lead to incorrect interpretation of results since it is now well established that enhancers can regulate genes over very large genomic distances through chromatin looping [13, 14].

The challenge now is to link disease-associated enhancers with the true genes that they regulate in order to determine the relevant cell types and the mechanism of regulation. Advances in sequencing, molecular biology and genome editing are now enabling us to answer these pivotal, "post-GWAS" questions [15]. Hi-C is a technique used to map interactions between distant DNA elements $[14,16]$. Its more recent derivative, Capture HiC (CHi-C), allows for high-depth characterisation of DNA interactions in loci of interest [17]. $\mathrm{CHi}-\mathrm{C}$ has been applied to gene promoters in multiple blood cell types [13] and to GWAS loci in diseases such as cancer [17-19] and autoimmune conditions [20]. HiChIP builds on $\mathrm{Hi}-\mathrm{C}$ by enriching for interactions that colocalise with an immunoprecipitated chromatin fraction (such as that marked by histone 3 lysine 27 acetylation, a hallmark of active chromatin) [21]. HiChIP was recently applied in primary $\mathrm{T}$ cells [22] and B cells, in the context of systemic lupus erythematosus (SLE) [23]. To empirically determine the function of GWAS SNPs, direct perturbation is now widely carried out using the CRISPRCas9 system, either through genome editing or chromatin activation/interference (CRISPRa/CRISPRi) of promoters and enhancers [24].

Here, we apply these technologies to annotate gene targets within known psoriasis GWAS loci utilising two relevant human cell lines: $\mathrm{HaCaT}$ (keratinocytes) and My-La (CD8+ T cells). We go on to experimentally characterise an intergenic psoriasis risk locus where the gene candidate, Krüppel-like factor 4 (KLF4), encodes a transcription factor with a range of relevant functions including skin barrier formation and immune signalling, but is situated more than $500 \mathrm{~kb}$ from the lead GWAS SNP. This study provides further evidence that KLF4 is a distal gene target in $9 \mathrm{q} 31.2$.

\section{Results}

\section{Capture Hi-C identified novel gene targets in psoriasis susceptibility loci}

We generated sequencing data for region $\mathrm{CHi}-\mathrm{C}$ experiments in duplicate in three conditions: (i) $\mathrm{HaCaT}$ unstimulated, (ii) $\mathrm{HaCaT}$ stimulated with IFN- $\gamma$ to represent the inflammatory psoriatic environment and (iii) My-La cells. Our overarching design targeted genetic regions associated with several immune-mediated diseases including psoriasis SNPs from multiple GWAS (see the "Methods" and Additional file 1, Table S1). We aimed for 10,000 and obtained an average of 8580 mapped Hi-C fragments (ditags) per bait fragment with a mean capture efficiency of $71 \%$ (Additional file 1, Table S2). Capture Hi-C Analysis of Genomic Organisation (CHiCAGO) was used to identify significant interactions for each cell type. Reproducibility was assessed firstly by observing the number of shared interactions between replicates and secondly through HiCRep [25] (Additional file 2, Fig. S1). The stratum-adjusted correlation coefficient (SCC) produced by HiCRep showed that all $\mathrm{HaCaT}$ samples were highly similar and were slightly more correlated by replicate rather than by condition. My-La samples were also highly correlated with each other, and less so with $\mathrm{HaCaT}$ cells (Additional file 2, Fig. S1B).

By integrating published ChIP-seq data, we found that other-end fragments interacting with the GWAS bait fragments were enriched in H3K27ac and H3K4me3 in related cell types NHEK and CD8+ naive T cells (Additional file 3, Fig. S2), suggesting that the GWAS loci preferentially interact with active regions such as enhancers and promoters. Other-end fragments in $\mathrm{HaCaT}$ cells were also enriched for the chromatin structural regulator CTCF, based on ChIP-seq in NHEK (Additional file 3, Fig. S2).

For the My-La cell line, we noted a large number of significant trans-interactions (CHiCAGO score $\geq 5$ ) spanning different chromosomes (3392/42,928 total interactions from all captured immune-mediated disease loci), and the majority of these ( $59 \%)$ mapped to interactions between two known translocated loci in My-La cells [26]. In light of this, the interactions were filtered to only include samechromosome interactions. We then filtered the $\mathrm{CHi}-\mathrm{C}$ interactions to include only those involving psoriasis GWAS loci; we had successfully targeted 104 lead GWAS SNPs at genome-wide significance and their associated proxy SNPs at $r^{2}>0.8$, corresponding to 907 HindIII bait fragments. Across the three capture experiments, we obtained an average of 6593 interactions (CHiCAGO score $\geq 5$ ) originating from targeted psoriasis fragments (Additional file 1, Table S2). The data were enriched for long-range interactions, with more than $75 \%$ of the significant interactions in the psoriasis loci spanning $>100 \mathrm{~kb}$ (Additional file 4, Fig. S3). The median interaction distances were $227 \mathrm{~kb}$ (HaCaT unstimulated), $234 \mathrm{~kb}$ (HaCaT stimulated) and 
$259 \mathrm{~kb}$ (My-La). The interaction distances in psoriasis loci were found to be significantly greater in My-La cells than in $\mathrm{HaCaT}$ cells (Kruskal-Wallis with Dunn's multiple comparisons test; $P<0.0001$ and $P=0.0011$ for $\mathrm{HaCaT}$ unstimulated and stimulated, respectively), suggesting cell-specific chromatin architecture.

To validate our $\mathrm{CHi}-\mathrm{C}$ data, we overlaid the interactions with a published expression quantitative trail locus (eQTL) dataset, in which the lead psoriasis SNP had been colocalised with the lead eQTL SNP in CD4+ T cells and monocytes [27]. We hypothesised that longdistance eQTL-gene promoter pairings would often implicate chromatin looping. The study reported 15 lead GWAS SNPs with 26 corresponding lead eQTL proxy SNPs, of which 16 proxies, representing 9 lead GWAS SNPs, overlapped baited fragments in our study. Eight of these proxies were captured within a HindIII fragment that contained, or was within $20 \mathrm{~kb}$ of, the eQTL gene itself. A further seven proxies were within, or adjacent to, fragments that showed evidence of interacting with the distal eQTL gene in our cell line $\mathrm{CHi}-\mathrm{C}$ data (CHiCAGO score $\geq 5$ ) (Additional file 5, Table S3). Only the most distant proxy, rs8060857, did not show any evidence of interacting with the eQTL gene (ZNF750, $720 \mathrm{~kb})$. Therefore, this is a strong evidence that our $\mathrm{CHi}-\mathrm{C}$ data can show links between distal functional GWAS SNPs and their target gene, even across non-matched cell types.

In all the cell lines, approximately $30 \%$ of the interactions occurred between the psoriasis bait fragment and a transcription start site (Ensembl 75). The total number of interacting gene targets was 442 in unstimulated $\mathrm{HaCaT}$ cells (Additional file 5, Table S4), 461 in stimulated $\mathrm{HaCaT}$ cells (Additional file 5, Table S5) and 650 in My-La cells (Additional file 5, Table S6), comprising a set of 839 genes. Of these, 288 gene targets (34.3\%) were shared between all cell types, whilst 58, 64 and 291 targets were unique in unstimulated $\mathrm{HaCaT}$, IFN- $\gamma$-stimulated $\mathrm{HaCaT}$ and My-La cells, respectively. Unstimulated and stimulated $\mathrm{HaCaT}$ cells shared a large proportion of their gene targets (355 targets; 77-80\%). Bait fragments that interacted with genes tended to interact with multiple promoter-containing fragments corresponding to different genes, a median of 2 fragments in $\mathrm{HaCaT}$ cells (unstimulated or stimulated) and 3 fragments in My-La cells, implicating between 2 and 3 genes (Additional file 6, Fig. S4), which is in line with previously reported findings [20, 22, 28].

We reasoned that gene targets with detectable expression in the same cell type would be more biologically relevant than those not expressed, so we performed RNA-seq (Additional file 7, Table S7) and determined the relative expression of genes interacting with psoriasis GWAS SNPs in each cell type (Additional file 5, Tables S4-S6) or overlapping GWAS bait fragments (Additional file 7, Table S8). Expressed genes interacting with
GWAS fragments included compelling psoriasis candidates such as IL23A, PTGER4, STAT3 and NFKBIZ. Importantly, we found that other-end fragments of $\mathrm{CHi}-\mathrm{C}$ interactions were significantly enriched for transcription start sites of expressed genes in the corresponding cell type (Additional file 3, Fig. S2). We searched for transcription factor binding motifs intersected by psoriasis SNPs interacting with active gene promoters using the tool SNP2TFBS [29] and discovered several significantly enriched factors, with the greatest enrichment found for REL, which is itself a candidate gene in the 2p16.1 locus [30] (Additional file 7, Table S9).

Stimulating $\mathrm{HaCaT}$ cells with IFN- $\gamma$ caused the differential expression of 535 genes (adjusted $P<0.10$ ): 88 downregulated and 447 upregulated (Additional file 7 , Table S10). Whilst the downregulated genes were not enriched for any biological pathways, the upregulated genes were enriched for 196 biological processes that included psoriasis-relevant GO terms such as "GO: 0045087 innate immune response" $\left(P=9.39 \times 10^{-20}\right)$, "GO:0034097 response to cytokine stimulus" $(P=7.32 \times$ $10^{-15}$ ) and "GO:0034340 response to type I interferon" $\left(P=1.08 \times 10^{-10}\right)$ (Additional file 7 , Table S11). Twelve of the differentially expressed genes overlapped a psoriasis capture bait fragment (Additional file 3, Table S11) and included ERAP1, ERAP2, IFIH1, RNF114, SOCS1 and $S T A T 2$. In addition, 12 differentially expressed genes were involved in bait-promoter interactions (Additional file 7, Table S12) and included candidates such as ICAM1, KLF4 and STAT3. However, the vast majority of these differentially expressed genes interacted similarly with the psoriasis-associated baits in both unstimulated and stimulated cells (CHiCAGO score $\geq 5$ ).

\section{Examples of $\mathrm{CHi}-\mathrm{C}$ interactions implicating target genes for psoriasis}

At the intergenic locus $9 \mathrm{q} 31.2$, the psoriasis association falls between two distant gene clusters where the suggested gene candidate was Krüppel-like factor 4 (KLF4) due to its relevant biological functions in differentiation and innate immunity [30]. Our $\mathrm{CHi}-\mathrm{C}$ data showed significant interactions (CHiCAGO score $\geq 5$ ) between the psoriasis association and the promoter of KLF4 in both unstimulated and stimulated $\mathrm{HaCaT}$ cells, over a distance of approximately $560 \mathrm{~kb}$ (Fig. 1a). In both conditions, the bait fragment chr9:110810592-110816598 interacted with KLF4 (CHiCAGO score $=6.75$ and 5.29 for unstimulated and stimulated cells, respectively) whilst in stimulated cells alone, a second bait fragment (chr9:110798319-110798738) also interacted, coinciding with a more than fivefold increase in the KLF4 expression ( $\mathrm{FC}=5.78$; adj. $P=4.26 \times$ $10^{-8}$ ). In My-La cells, a similar conformation was observed; however, the interactions did not coincide with the fragment containing the gene itself. Furthermore, the KLF4 


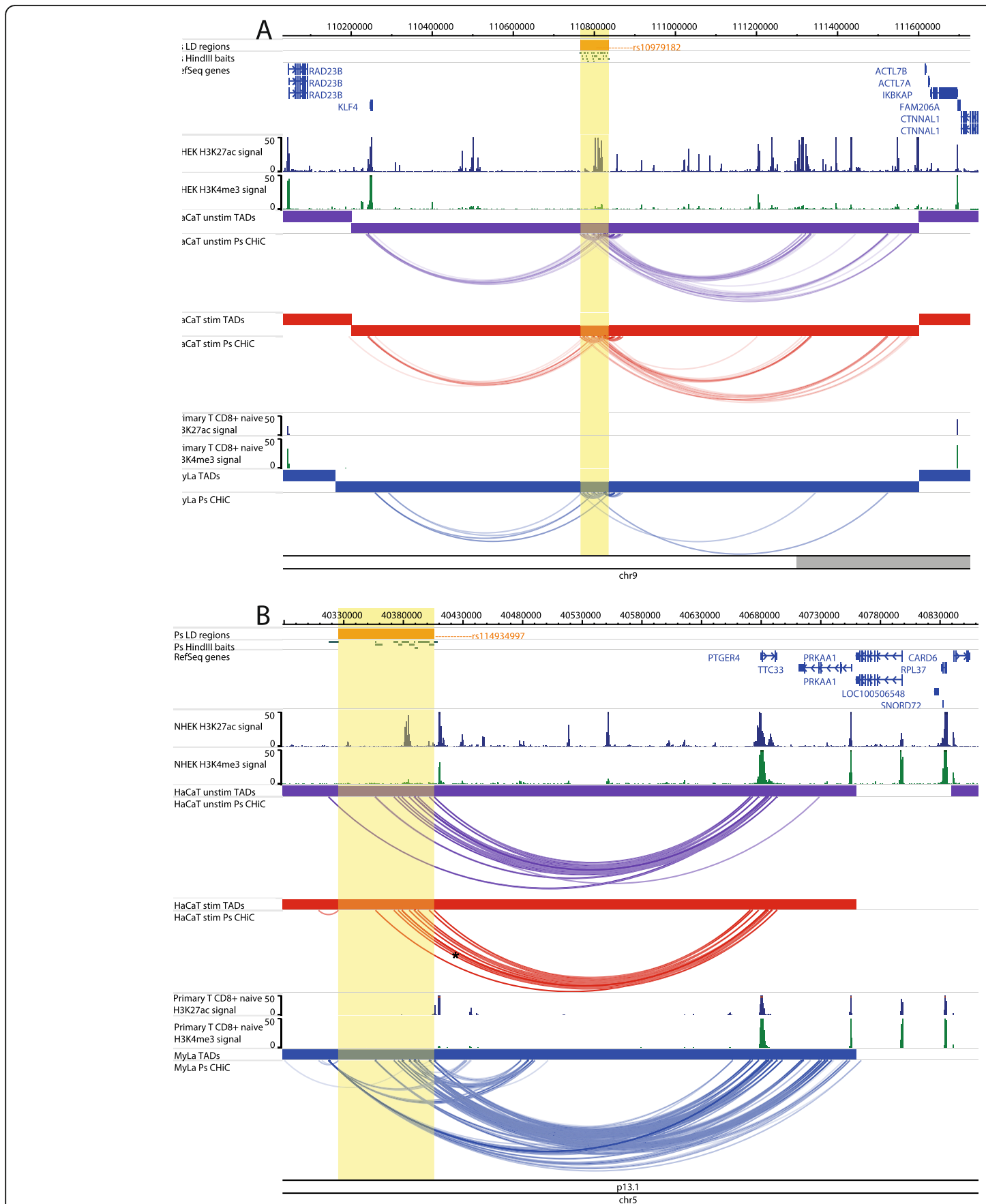

Fig. 1 Examples of $\mathrm{CHi}-\mathrm{C}$ Interactions implicating nearest/reported genes. Interactions are shown in the 9q31.2 (KLF4) locus (a) and the 5 p13.1 (PTGER4) locus (b). The tracks include psoriasis (Ps) LD blocks as defined by SNPs in $r^{2}>0.8$ with the index SNP, baited Hindlll fragments, RefSeq genes (NCBI), H3K27ac and H3K4me3 P value signal in NHEK (ENCODE) and CD8+ primary naive T cells (Roadmap Epigenomics), TADs (shown as bars) and $\mathrm{CHi}-\mathrm{C}$ interactions significant at CHiCAGO score $\geq 5$ (shown as arcs) in three conditions: unstimulated HaCat cells (purple), HaCaT cells stimulated with IFN-y (red) and My-La cells (blue). The highlighted region indicates the psoriasis LD block. The figure was made with the WashU Epigenome Browser, GRCh37/hg19 [31]

expression was not detected by RNA-seq in My-La cells, suggesting a cell type-specific mechanism (Additional file 7, Table S7). In all cell types, long-range interactions also stretched from the psoriasis locus to the telomeric side of the gene desert but fell short of the nearest gene on that side $(A C T L 7 B)$ by approximately $35 \mathrm{~kb}$.

At the 5 p13.1 locus, the psoriasis SNPs are similarly intergenic [32], but the nearest gene PTGER4 has been 
shown to be a strong candidate for other autoimmune diseases at this locus [22]. Our $\mathrm{CHi}-\mathrm{C}$ data showed interactions (CHiCAGO score $\geq 5$ ) between multiple psoriasis-associated fragments and PTGER4 over approximately $300 \mathrm{~kb}$ to the other end of the TAD, a finding that was robust in all cell types (Fig. 1b). PTGER4 expression was detected by RNA-seq in all cell types (Additional file 7, Table S7). In My-La cells, interactions also stretched to the promoters of TTC33, which was expressed in all cell types, and RPL37, for which expression was not detected in any cell type.

At the 2 p15 locus, the psoriasis association tagged by rs10865331 was originally assigned to the nearest gene B3GNT2; however, the $\mathrm{CHi}-\mathrm{C}$ interactions skipped B3GNT2 ( $120 \mathrm{~kb}$ upstream) and instead implicated the promoter of copper metabolism domain containing 1 (COMMD1), a gene involved in NFkB signalling, over approximately $435 \mathrm{~kb}$ upstream (Fig. 2a) [30, 33]. This interaction occurred in stimulated $\mathrm{HaCaT}$ cells and MyLa cells, and COMMD1 expression was detected by RNA-seq in all cell types. B3GNT2 expression was also detected in all cell types (Additional file 7, Table S7).

At the 1p36.23 locus, the association tagged by rs11121129 is closest to SLC45A1 and was originally assigned to multiple putative gene targets [30]. However, the $\mathrm{CHi}-\mathrm{C}$ data showed interactions (CHiCAGO score $\geq$ $5)$ between the psoriasis LD block and the promoter of ERBB receptor feedback inhibitor 1 (ERRFI1), an important regulator of keratinocyte proliferation and differentiation, in both unstimulated and stimulated $\mathrm{HaCaT}$ cells (Fig. 2b). This interaction was not observed in My-La cells, and moreover, ERRFI1 expression was detected in $\mathrm{HaCaT}$ cells (unstimulated and stimulated) but not in My-La cells (Additional file 7, Table S7). An interaction between the psoriasis association and the promoter of SLC45A1 was also observed in stimulated, but not in unstimulated, HaCaT cells (Fig. 2b); however, SLC45A1 expression was not detected by RNA-seq in any of the cell lines (Additional file 5, Table S5).

At the 6p22.3 locus, the psoriasis signal tagged by rs4712528 is intronic to CDKAL1, and there were 11 psoriasis-associated intronic fragments that also interacted with the CDKAL1 promoter in My-La cells (Fig. 3a); CDKAL1 expression was detected in all cells. However, there were also long-range interactions (CHiCAGO score $\geq 5$ ) between psoriasis-associated fragments and SOX4 over $950 \mathrm{~kb}$ in all cell types (Fig. 3a). SOX4 is a compelling gene candidate with roles in IL17A production and skin inflammation in mice [34]; here, SOX4 expression was detected in $\mathrm{HaCaT}$ cells but not in My-La cells (Additional file 7, Table S7).

At the 1q21.3 locus, multiple risk SNPs are located at the late cornfield envelope (LCE) gene cluster in the epidermal differentiation complex (EDC). One of the associations in this locus is a $32-\mathrm{kb}$ deletion that removes the $L C E 3 B$ and $L C E 3 C$ genes [30, 35, 36]. The $\mathrm{CHi}-\mathrm{C}$ data showed multiple, robust interactions between the psoriasis-associated regions at the LCE genes, including from within the 32-kb LCE3C/B-del region, and genes downstream in the EDC that included IVL, LOR, PRR9 and SPRR genes, over a distance of $\sim 600 \mathrm{~kb}$ (Fig. 3b). Of these genes, IVL interacted with psoriasis baits in unstimulated but not in stimulated $\mathrm{HaCaT}$ cells, and its expression decreased upon stimulation ( $\mathrm{FC}=0.40$; adj. $P=0.0139$ ). The coding genes directly interacting with fragments within the $32-\mathrm{kb}$ deletion were LCE3A, PRR 9, LELP1, SPRR2B and SPRR2C. Of these, only the expression of proline-rich region 9 (PRR9) was detected, in $\mathrm{HaCaT}$ cells but not in My-La cells (Additional file 7, Table S7). PRR9 was previously shown to be upregulated in psoriatic plaques and induced by IL17A and so may be an important distal gene target in this locus [37].

The $9 q 31.2$ psoriasis risk locus forms long-range interactions with KLF4 and harbours likely regulatory variants

We focused our attention on the large intergenic locus at 9q31.2, which has not previously been characterised in psoriasis, to our knowledge. The candidate gene, Krüppellike factor 4 (KLF4), encodes a transcription factor with a range of relevant functions including skin barrier formation [38] and immune signalling [39] but is situated more than $500 \mathrm{~kb}$ from the lead GWAS SNP rs10979182 [30]. The $\mathrm{CHi}-\mathrm{C}$ experiment showed long-range interactions between the psoriasis-associated SNPs and KLF4 (Fig. 1a) [30]. KLF4 expression was also upregulated by IFN- $\gamma$, suggesting that it may be an important player within an inflammatory environment. We wanted to prioritise regulatory variants in 9q31.2 and determine if any functional enhancer-promoter relationship existed between the SNPs and KLF4 or other, distal, genes in the locus.

First, we characterised the psoriasis-associated SNPs in $9 \mathrm{q} 31.2$ by mining publicly available epigenetic datasets and tools. There are ninety variants in tight $\mathrm{LD}\left(r^{2}>0.8\right)$ with the lead GWAS SNP rs10979182 (1KG Phase 3 European) (Fig. 4a); several of which intersect modified histone marks (H3K4me1 and H3K27ac) in several cell types from ENCODE, corresponding with four putative enhancer elements overlapping H3K4me1 and H3K27ac occupancy (Fig. 4b). In primary human keratinocyte (NHEK) cells, enhancer histone marks were most prominent in enhancers 2-4 (Fig. 4c). The SNPs also overlap DNase hypersensitivity sites and transcription factor binding sites (Fig. 4c) that correspond with enhancer elements in NHEK according to ChromHMM [43].

No eQTLs were identified in the set according to Haploreg v4.1. RegulomeDB identified rs6477612, situated within the third putative enhancer, as the SNP with the highest putative regulatory potential with a score of $2 \mathrm{a}$. rs6477612 is in tight LD $\left(r^{2}=0.92\right.$, 1KG EUR) with rs10979182 and was located within the HindIII fragment 

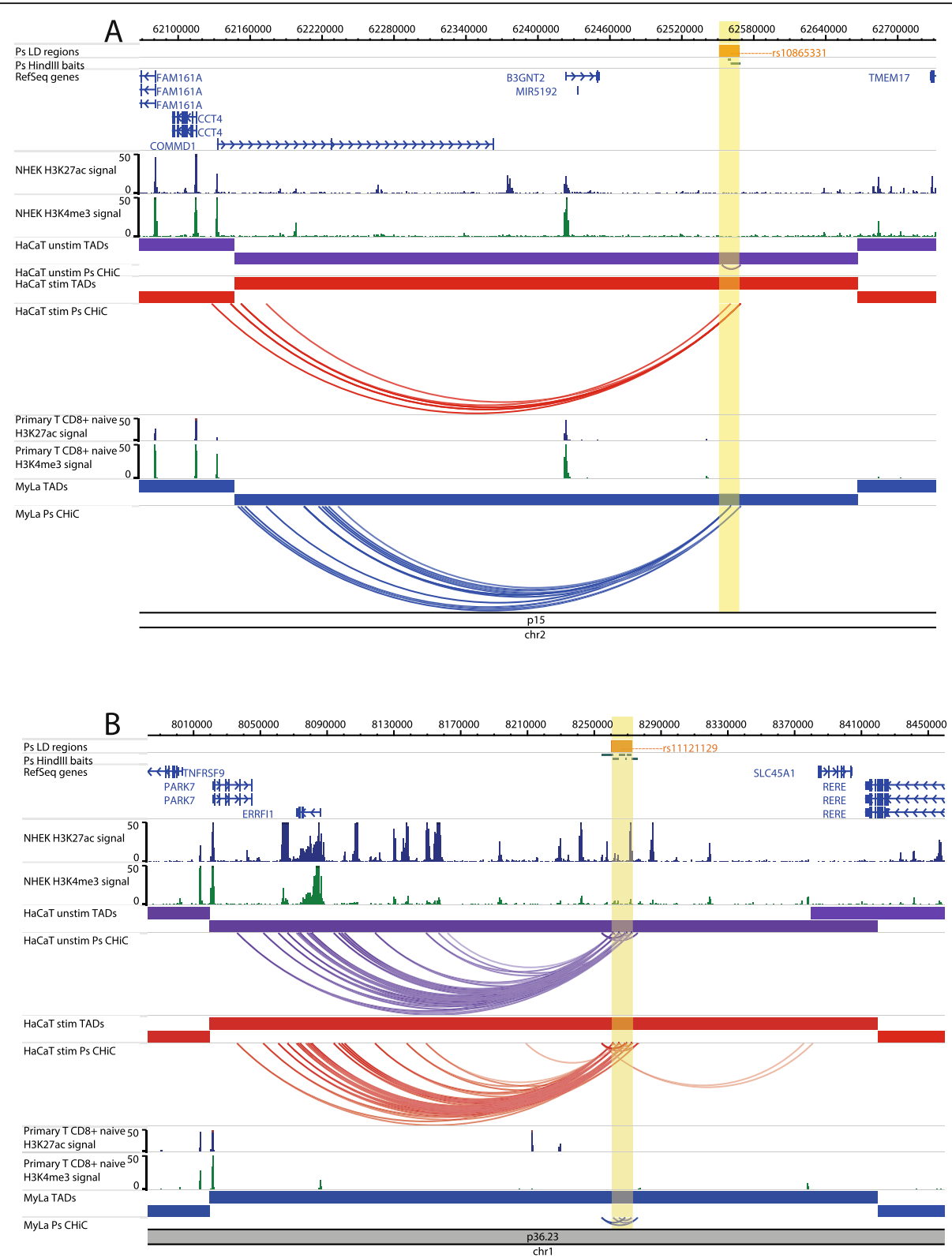

Fig. 2 Examples of CHi-C interactions in gene deserts implicating distal/novel genes. Interactions are shown in the 2p15 (B3GNT2) locus (a) and the 1p36.23 (RERE, SLC45A1, ERRFI1, TNFRSF9) locus (b). The tracks include psoriasis (PS) LD blocks as defined by SNPs in $r^{2}>0.8$ with the index SNP, baited Hindlll fragments, RefSeq genes (NCBI), H3K27ac and H3K4me3 P value signal in NHEK (ENCODE) and CD8+ primary naive T cells (Roadmap Epigenomics), TADs (shown as bars) and CHi-C interactions significant at CHiCAGO score $\geq 5$ (shown as arcs) in three conditions: unstimulated HaCat cells (purple), HaCaT cells stimulated with IFN- (red) and My-La cells (blue). The highlighted region indicates the psoriasis LD block. The figure was made with the WashU Epigenome Browser, GRCh37/hg19 [31]

found to interact with KLF4 in $\mathrm{HaCaT}$ cells in our $\mathrm{CHi}$ C data (chr9:110810592-110816598; hg19), making it a prioritised SNP of interest.

HiChIP data suggested that the interactions between KLF4 and psoriasis SNPs are active in $\mathrm{HaCaT}$ cells, but not in My-La cells

As a complementary approach to $\mathrm{CHi}-\mathrm{C}$, we used the recently developed HiChIP method to identify H3K27ac- mediated interactions in our cell lines. In $\mathrm{HaCaT}$ cells, there was a H3K27ac peak at the KLF4 promoter that interacted with several regions across the gene desert including psoriasis-associated enhancers 3 and 4 and at the previously published interacting region from the breast cancer study in both unstimulated and stimulated $\mathrm{HaCaT}$ cells (Fig. 5) [17]. The H3K27ac peaks at the psoriasis SNPs also interacted with several other putative enhancers within the gene desert, but did not interact 

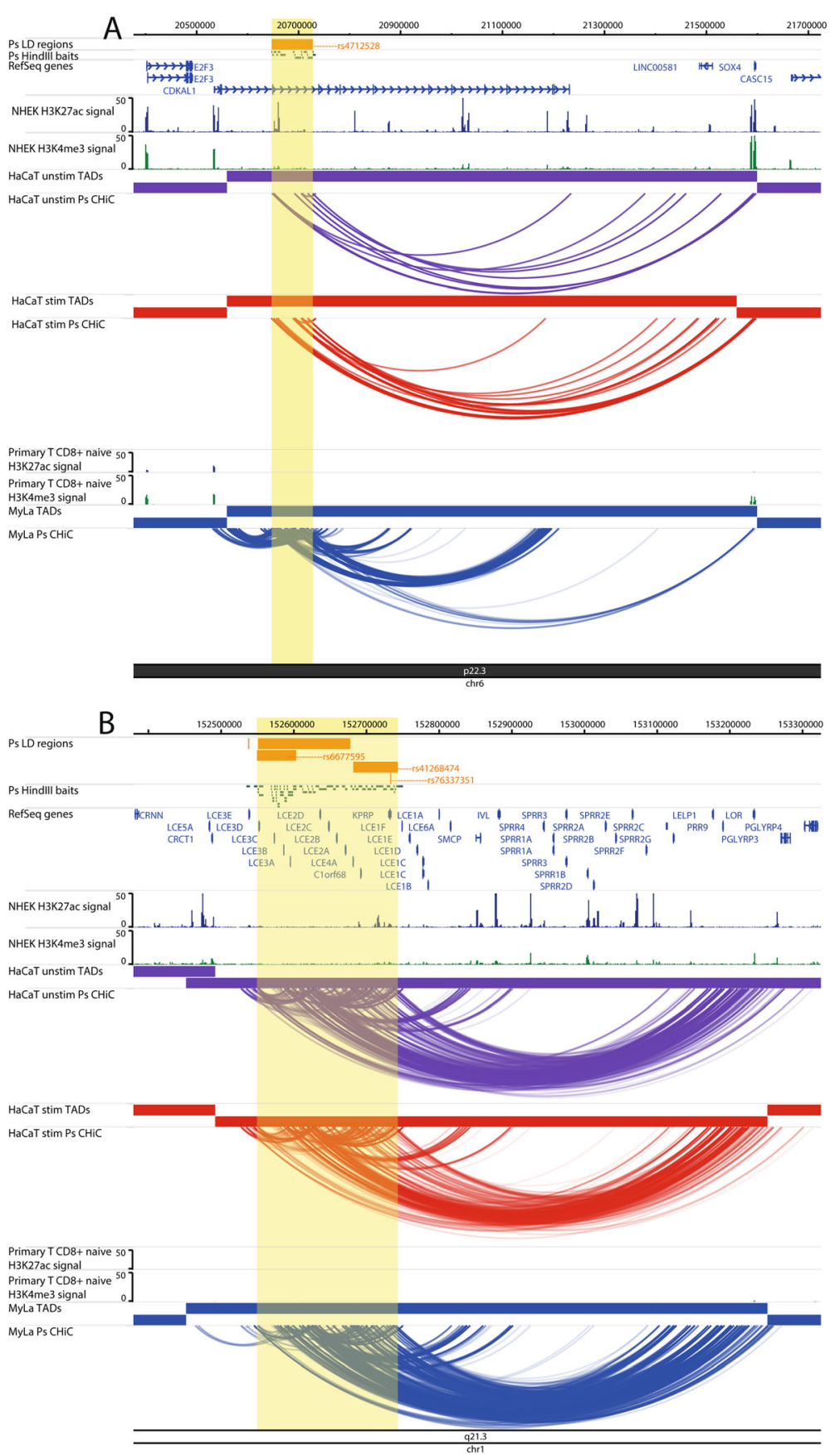

Fig. 3 Examples of $\mathrm{CHi}-\mathrm{C}$ interactions adding complexity to a locus. Interactions are shown in the 6p22.3 (CDKAL1) locus (a) and the 1q21.3 ( $L C E 3 B, L C E 3 C$ ) locus (b). The tracks include psoriasis (PS) LD blocks as defined by SNPs in $r^{2}>0.8$ with the index SNP, baited Hindlll fragments, RefSeq genes (NCBI), H3K27ac and H3K4me3 $P$ value signal in NHEK (ENCODE) and CD8+ primary naive T cells (Roadmap Epigenomics), TADs (shown as bars) and $\mathrm{CHi}-\mathrm{C}$ interactions significant at CHiCAGO score $\geq 5$ (shown as arcs) in three conditions: unstimulated HaCat cells (purple), HaCaT cells stimulated with IFN- $\gamma$ (red) and My-La cells (blue). The highlighted region indicates the psoriasis LD block. The figure was made with the WashU Epigenome Browser, GRCh37/hg19 [31]

with other gene targets, mirroring the $\mathrm{CHi}-\mathrm{C}$ architecture (Fig. 5). In contrast, there was a lack of H3K27ac peaks in My-La cells in 9q31.2 and, correspondingly, no significant HiChIP interactions. This lack of H3K27ac occupancy indicates a differential activation state in this region between $\mathrm{HaCaT}$ and My-La cells.

We observed an increase in the number and strength of H3K27ac peaks in the 9q31.2 TAD (chr9:110202281- 


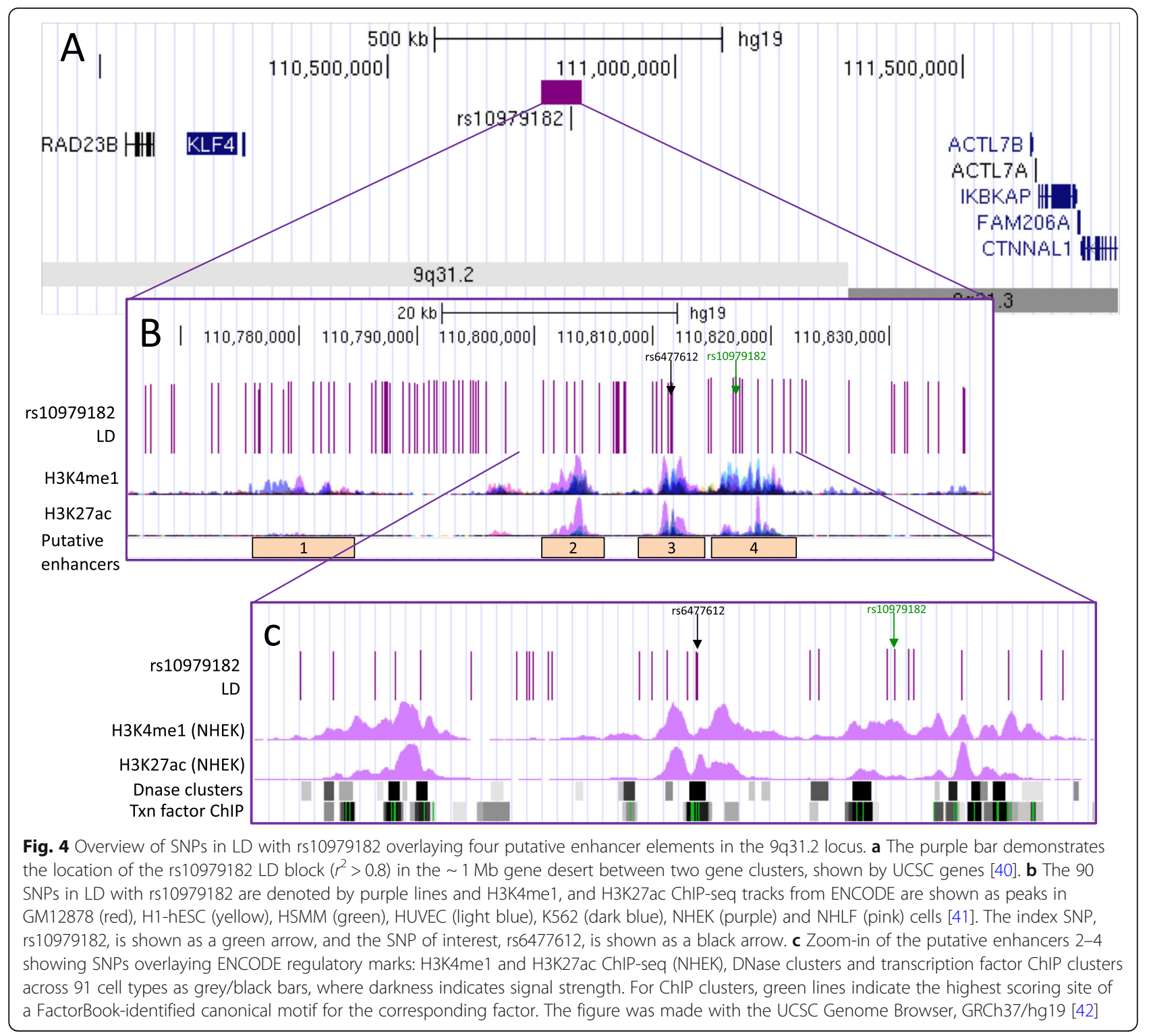

111602280) in stimulated $\mathrm{HaCaT}$ cells compared with unstimulated $\mathrm{HaCaT}$ cells (Fig. 5). The number of peaks increased from 60 to 77, and there was a significant increase in the median peak signal from $\sim 5.3$ to $\sim 9.5$ in shared peaks $(P<0.0001$, Wilcoxon matched-pairs signed-rank test). This also corresponded with an over 5 -fold upregulation of gene expression upon IFN- $\gamma$ stimulation in $\mathrm{HaCaT}$ cells $\left(\mathrm{FC}=5.78\right.$; adj. $\left.P=4.26 \times 10^{-8}\right)$. Combined, this suggests that inflammatory stimulation of $\mathrm{HaCaT}$ cells causes upregulation of KLF4 that is mediated, or at least accompanied, by increased enhancer activity in 9q31.2.

\section{C-qPCR supplemented HiChIP/CHi-C findings in the 9q31.2 locus}

We used 3C-qPCR in an effort to confirm the interaction between the psoriasis-associated putative enhancer 3 (rs6477612) and KLF4 and to further prioritise regulatory SNPs. Our 3C experiment utilised both the enhancer and the KLF4 gene as focus anchors, in both $\mathrm{HaCaT}$ and $\mathrm{My}-\mathrm{La}$ cell lines. The enhancer-focused $3 \mathrm{C}$ experiment identified interaction peaks with regions approximately $2.5 \mathrm{~kb}$ and 8.7 $\mathrm{kb}$ downstream of KLF4 in My-La and with the downstream 8.7-kb region alone in $\mathrm{HaCaT}$ (Additional file 8, Fig. S5).

The KLF4-focused 3C experiment showed that KLF4 significantly interacted with several intergenic psoriasisassociated fragments, including the fragment containing the third putative enhancer (rs6477612), in $\mathrm{HaCaT}$ cells, but not in My-La cells (Additional file 9, Fig. S6). This corroborates the $\mathrm{CHi}-\mathrm{C}$ data, which showed a more robust interaction between the enhancer and the KLF4 gene in $\mathrm{HaCaT}$ cells (Fig. 1a). A positive control interaction linking a distal breast cancer-associated locus with KLF4 [17, 18] 




demonstrated the strongest interaction with the KLF4 promoter region in both cell types (Additional file 9, Fig. S6).

Taken together, the $3 \mathrm{C}$ results confirm a close spatial proximity between the psoriasis-associated SNPs and KLF4 in 9q31.2. However, there is no clear peak of interaction among the LD block that would implicate some SNPs over others. In addition, stronger interactions were seen between KLF4 and regions further upstream in the gene desert, which correlates with previous $\mathrm{CHi}-\mathrm{C}$ findings in breast cancer cells [17] and Hi-C findings in NHEK cells [14] (illustrated in Additional file 10, Fig. S7).

\section{ChIP-qPCR confirmed the presence of regulatory histone modifications in $9 \mathrm{q} 31.2$ in $\mathrm{HaCaT}$ cells}

We performed ChIP-qPCR of the histone marks H3K4me1 and H3K27ac to confirm the cell type specificity of enhancer activity within the KLF4-interacting psoriasis loci. Primers were designed to target 150-200-bp regions encompassing predicted peaks of H3K27ac occupancy in the four putative enhancers identified from ENCODE data (NHEK). H3K4me1 and H3K27ac occupancy was detected at all tested loci in HaCaT and My-La cells (Fig. 6a). However, occupancy was significantly increased in $\mathrm{HaCaT}$ cells with an enrichment of both H3K4me1 and H3K27ac at enhancer 3 (H3K4me1 $P=0.0372$, H3K27ac $P<0.0001$ ) and H3K27ac at enhancer $4(P<0.0001)$ in $\mathrm{HaCaT}$ cells in comparison with My-La cells (Fig. 6a). Stimulation of $\mathrm{HaCaT}$ cells with IFN- $\gamma$ had little effect on the occupancy of H3K4me1 or $\mathrm{H} 3 \mathrm{~K} 27 \mathrm{ac}$ at the regions tested within the enhancers, or at a region tested at the KLF4 promoter (Fig. $6 \mathrm{~b}$ ).

To determine the potential effects of the risk or protective allele of rs6477612, a SNP of interest within 


\section{H3K4me1}

\section{A}

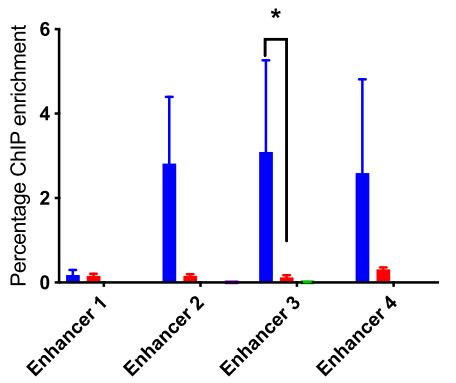

B



C

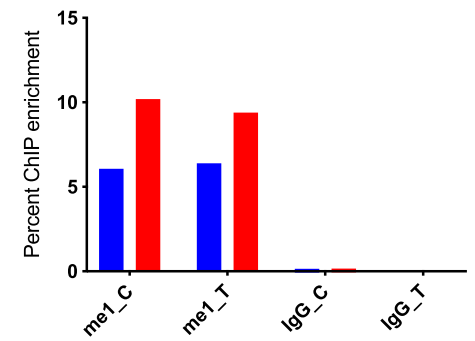

H3K27ac


Fig. 6 ChIP-qPCR for modified histone marks H3K4me1 and H3K27ac in 9q31.2. a Enhancer peaks defined by H3K27ac binding in ENCODE NHEK data were targeted in HaCaT cells (blue columns) and My-La cells (red columns). b Enhancer peaks were targeted in unstimulated (blue) and stimulated (red) $\mathrm{HaCaT}$ cells. The graphs show the mean ChIP enrichment of triplicate ChIP libraries \pm SD, and samples with no antibody are consistently included for comparison, although they are often too low to be visible. To identify differential ChIP enrichment, 2-way ANOVA tests were performed in GraphPad prism using Sidak's multiple comparisons test. Asterisks denote adjusted $P<0.05$. c Allele-specific ChIP-qPCR for H3K27ac and H3K4me1 at rs6477612 in NHEK cells. Chromatin from two separate pools of NHEK cells, each comprising cells from three individual donors, was immunoprecipitated with H3K27ac (27 ac), H3K4me1 (me1) or non-specific lgG antibody (lgG), and qPCR was conducted using a TaqMan genotyping assay for rs6477612 detecting C (risk) or T (protective) alleles. Percentage ChIP enrichment was calculated by comparing the signal for each allele in the immunoprecipitated DNA with the signal for each allele in the input DNA for each of the two samples

enhancer 3, we performed allele-specific ChIP at rs6477612 for H3K4me1 and H3K27ac in two pools of NHEK cells. However, there was no discernible difference in H3K4me1 or H3K27ac occupancy at the risk (C) or protective (T) allele of rs6477612 (Fig. 6c).

In summary, by combining the HiChIP, $\mathrm{CHi}-\mathrm{C} 3 \mathrm{C}$ and ChIP evidence, we could determine that the psoriasisassociated enhancer region interacts with KLF4 in both My-La and $\mathrm{HaCaT}$ but is only active in $\mathrm{HaCaT}$ cells. Enhancer activity in 9q31.2 is increased after IFN- $\gamma$ stimulation, correlating with an increase in KLF4 gene expression, although we were unable to detect increases in H3K27ac occupancy at the tested psoriasis-associated enhancer regions.
CRISPR activation suggested that the psoriasis-associated enhancer elements regulate KLF4 expression in 9q31.2 We employed CRISPRa in 9q31.2 to determine whether activating the psoriasis-associated enhancers could impact on gene expression (KLF4 or other, more distal genes), implicating a functional role for the long-range interactions. Pools of single-guide RNA (sgRNA) targeting SNPs within the four psoriasis-associated enhancers were introduced into $\mathrm{HaCaT}$ cells stably expressing the CRISPR activator dCas9-P300 (see Additional file 11, Fig. S8 for overview of sgRNA locations). All four pools of sgRNA increased the KLF4 expression in comparison with the control, scrambled sgRNA; this increase was statistically significant (after multiple testing correction) 
at enhancer $3(P=0.0143)$ and enhancer $4(P=0.0183)$ (Fig. 7). Pool 3, targeting enhancer 3 containing rs6477612, had the greatest impact with a 2.2-fold increase in the KLF4 expression. To a lesser extent, Ikap$\mathrm{paB}$ kinase complex-associated protein (IKBKAP) to the telomeric end of the gene desert was also subtly but significantly upregulated by approximately 1.2 -fold in cell lines containing sgRNA pool 1 in comparison with the scrambled sgRNA $(P=0.0372)$. We found that FAM206A and CTNNAL1 were not significantly affected by CRISPRa (Fig. 7). The remaining two genes, ACTL7A and $A C T L 7 B$, were not detectable in any $\mathrm{HaCaT}$ cell line, transduced or otherwise (for $A C T L 7 A$, all Ct values $\geq 33.4$ and for $A C T L 7 B$, all Ct values $\geq 34.1$ ).

To determine the transcriptome-wide effects of activating the psoriasis-associated enhancers in 9q31.2, RNA-seq was performed on the $\mathrm{HaCaT}$ dCas9-P300 cells expressing the sgRNA in pool 3 (putative enhancer 3 ) and compared with cells expressing the scrambled sgRNA (Additional file 12 Table S13). In line with the qPCR experiment, RNA-seq revealed an approximately 3 -fold increased expression of KLF4 in the pool 3 cells $(\mathrm{FC}=2.92$; adj. $P=0.0546)$ and an approximately 1.2 fold increase in IKBKAP, CTNNAL1 and FAM206A expression, but these were not significant (IKBKAP FC=
1.26, adj. $P=0.7331 ; C T N N A L 1 \mathrm{FC}=1.23$, adj. $P=0.68$; FAM206A $\mathrm{FC}=1.24$, adj. $P=0.82$ ). Therefore, KLF4 is the only candidate in this locus with convincing evidence for cis-regulation by the targeted enhancers.

The RNA-seq analysis showed that there were an additional 236 differentially expressed genes in the CRISPRa experiment (adjusted $P \leq 0.10$ ), 128 upregulated and 108 downregulated (Additional file 12 Table S13). Importantly, CRISPRa of the psoriasis-implicated enhancer in this keratinocyte cell line not only resulted in an increase in KLF4 expression, but a differential expression of 3 keratin genes (keratin 4, 13 and 15), confirming the importance of this enhancer and the KLF4 gene in skin cell function. Keratin 4 was the most differentially expressed gene in our data with keratin 15 being the 6th most differentially expressed. Previous studies also demonstrated a differential impact of KLF4 on keratin gene regulation [44-46].

Confirming the importance of KLF4 in skin cells and validating previous findings of differential gene expression with KLF4 stimulation, differential genes also included EREG (an epidermal growth factor), MMP13 (extracellular matrix protein gene) and CLDN8 (claudin 8; important in epithelium tight junctions). We also demonstrated a $\sim 10$-fold reduction in the expression of




ALPG, from a family of alkaline phosphatases showing the largest fold change in a previous KLF4 overexpression study [44] (Additional file 12 Table S13). The upregulated genes were enriched in several biological pathways according to the GAGE pathway analysis, of which, the most significant related to RNA processing (Additional file 12, Table S14). According to the STRING database, differential genes were enriched in a number of relevant pathways including apoptosis and response to cell stress, emphasising the role that KLF4 plays in cell cycle regulation and supporting previous findings demonstrating that over-expressing KLF4 leads to G1/S cell cycle arrest [47] (Additional file 12, Table S15).

To confirm if the differential genes in the CRISPRa experiment were regulated by KLF4, we used a recently published tool that predicts and ranks transcriptional regulators of gene sets, Lisa [48]. The tool predicted that indeed the gene set was significantly regulated by KLF4 in keratinocytes, breast epithelium and skin fibroblasts. However, KLF4 was ranked number 104 of all the transcriptional regulators (Additional file 12, Table S16). We speculate that the CRISPRa experiment may yield many differentially expressed genes that are not directly regulated by KLF4, but rather are involved in the further downstream cascade of altered gene regulation.

Taken together, the chromatin interaction data coupled with the CRISPRa experiment allow for prioritisation of likely causal variants in 9q31.2 (Additional file 12 Table S17). From the 90 variants in LD with the index SNP, rs10979182, four variants interacted with the likely causal gene KLF4 in both the CHi-C and HiChIP data and overlapped H3K27ac peaks (HaCaT cells): rs60082362, rs55975335, rs6477612 and rs6477613. These variants are all located in the CRISPRa pool 3, which marginally had the greatest impact on the KLF4 expression.

\section{Discussion}

Genetic predisposition is the largest known risk factor for psoriasis. The GWAS era has provided a wealth of genetic loci associated with psoriasis, and yet, if we are to exploit these data fully, for clinical benefit with new and more effective therapies, there is a requirement for a better understanding of their biological significance. Recent functional studies have used sophisticated post-GWAS technologies to assign gene targets, cell types and functional mechanisms in the loci associated with other, related complex conditions [20, 49-51]. Here, we have combined these technologies for the first time focused on investigation into the functional genomics of psoriasis.

Our study provides findings that are complementary to previously published data. As an example, our data in cell lines demonstrates a long-range interaction between psoriasis-associated SNPs and PTGER4 in the intergenic psoriasis risk locus at $5 \mathrm{p} 13.3$, similar to published, promoter $\mathrm{CHi}-\mathrm{C}$ data in several other primary cell types, including psoriasis-relevant cells such as macrophages, monocytes, CD4+ T cells, CD8+ T cells and neutrophils [13]. Recently, HiChIP again demonstrated how this locus forms functional enhancer interactions with PTGER4 [22]. We also demonstrate how chromatin conformation data can provide evidence for suspected causal gene targets or provide support for regions that show an eQTL to a putative causal gene.

In the intergenic 9q31.2 psoriasis risk locus, we implicate $K L F 4$ as the likely causal psoriasis risk gene in this region by its functional interaction with the psoriasis GWAS variants. By incorporating RNA-seq analysis, we demonstrate that activating the disease implicated enhancers also activates downstream pathways of KLF4, showing how perturbation of a regulatory sequence and modest effect on transcription factor expression can have a profound effect on downstream gene targets in disease. KLF4 protein contains both an activation and repression domain and is known to either upregulate or downregulate pathways in a tissue- and contextdependent manner; therefore, its role in disease is likely to be complex [52], but it has already been shown by others to be dysregulated in psoriatic plaques [53]. Whilst our study was limited in its ability to identify a single causal variant, our findings did prioritise four variants from 90 in LD with the lead SNP thereby providing a solid basis for further follow-up using novel technologies such as fine-tuned CRISPR experiments.

\section{Conclusions}

In conclusion, we provide evidence for putative gene targets in psoriasis risk loci, supporting assigned candidates and in some regions suggesting novel candidates. We also focus on a specific risk locus, and by moving from associated variants to gene, cell type mechanism and pathway, we demonstrate how KLF4 is a likely gene target of the GWAS association in 9q31.2. This investigative pathway is applicable to all GWAS studies and helps make the next pivotal step towards patient benefit and clinical translation.

\section{Methods \\ Cell culture}

$\mathrm{HaCaT}$ keratinocyte cells were obtained from Addexbio (T0020001); these are in vitro spontaneously transformed keratinocytes from histologically normal skin. Cells were cultured in high-glucose Dulbecco's modified Eagle's medium (DMEM) supplemented with 10\% foetal bovine serum (FBS) and penicillin-streptomycin (Thermo Fisher Scientific, final concentration $100 \mathrm{U}$ penicillin, $0.1 \mathrm{mg}$ streptomycin $/ \mathrm{mL}$ ). For $\mathrm{HaCaT}$ stimulation experiments, the media were supplemented with $100 \mathrm{ng} / \mathrm{mL}$ recombinant human IFN- $\gamma$ (285-IF-100; R\&D Systems) and cells incubated for $8 \mathrm{~h}$ prior to harvest. 
Pools of adult normal human epidermal keratinocytes (NHEK) were obtained from PromoCell (C-12006) and cultured in Keratinocyte Growth Medium 2 (PromoCell) supplemented with $0.06 \mathrm{mM} \mathrm{CaCl}_{2}$.

My-La CD8+ cells were obtained from Sigma-Aldrich (95051033). These cells are cancerous human T lymphocytes derived from a patient with mycosis fungoides. Cells were cultured in Roswell Park Memorial Institute (RPMI) 1640 medium supplemented with $10 \% \mathrm{AB}$ human serum (Sigma Aldrich), $100 \mathrm{U} / \mathrm{mL}$ recombinant human IL-2 (Sigma-Aldrich) and penicillin-streptomycin (final concentration $100 \mathrm{U}$ penicillin, $0.1 \mathrm{mg}$ streptomycin $/ \mathrm{mL}$ ).

Lenti-X 293T cells were obtained from Takara Biosciences (632180). These cells are a sub-clone of the human embryonic kidney (HEK) cell line and are optimised for viral protein production. 293T cells were cultured in DMEM high glucose supplemented with $10 \%$ FBS and penicillin streptomycin (final concentration $100 \mathrm{U}$ penicillin, $0.1 \mathrm{mg}$ streptomycin $/ \mathrm{mL}$ ).

\section{Cell crosslinking for chromatin-based experiments}

$\mathrm{HaCaT}$ and NHEK cells were crosslinked for $10 \mathrm{~min}$ in $1 \%$ formaldehyde, and the reaction was quenched with $0.135 \mathrm{M}$ glycine. The crosslinked cells were pelleted and washed in PBS and the supernatant removed. Cells were snap frozen on dry ice and stored at $-80^{\circ} \mathrm{C}$. My-La cells were crosslinked for $10 \mathrm{~min}$ in 1\% (ChIP, HiChIP) or $2 \%$ (Hi-C, 3C) formaldehyde. The reaction was quenched with $0.135 \mathrm{M}$ glycine, the supernatant removed and the cells snap frozen on dry ice and stored at $-80^{\circ} \mathrm{C}$.

\section{Capture Hi-C}

For $\mathrm{CHi}-\mathrm{C}$, RNA baits were designed to target all known non-MHC psoriasis risk loci, defined by one or more independent SNPs associated with psoriasis in GWAS (Additional file 1, Table S1). The total number of SNPs included was 107 (59 associated with Europeans, 42 with Chinese, and 6 associated with both European and Chinese cohorts) corresponding with 68 loci. The baits were selected to target HindIII fragments that overlapped with the linkage disequilibrium (LD) block in each locus, defined by SNPs in $r^{2}>0.8$ with the lead SNP (1000 Genomes Phase 3 release, European). Due to sequence restraints, baits could not be designed for the $1 \mathrm{p} 36.11$ (rs7552167, rs4649203) [30, 54] and 1q31.1 (rs10789285) [32] loci; therefore, in total, there were 104 SNPs corresponding with 66 psoriasis risk loci in the final capture library (907 HindIII fragments).

The psoriasis baits were designed as part of a capture library targeting multiple GWAS loci across several immune-mediated diseases: juvenile idiopathic arthritis, asthma, psoriatic arthritis, rheumatoid arthritis and systemic sclerosis. The majority of these baits were included in our previous region $\mathrm{CHi}-\mathrm{C}$ experiment [20]. This initial capture library was applied to My-La Hi-C libraries, whereas a slightly updated capture library, which included some additional baits, was applied to $\mathrm{HaCaT} \mathrm{Hi-C} \mathrm{librar-}$ ies. The two different baitmaps included the same psoriasis regions, and results from non-psoriasis loci are not described in the present study. In addition, a control locus, which represents a well-characterised region of long-range interactions, $H B A$, was also included (174.57 kb genomic, 26 restriction fragments, $6.71 \mathrm{~kb} /$ restriction fragment). Each 120-bp bait was targeted to within 400 bp of a HindIII fragment end, comprised $25-65 \%$ GC content and contained fewer than three unknown bases. The baits were synthesised by Agilent Technologies.

$\mathrm{CHi}-\mathrm{C}$ libraries were generated in biological duplicate for $\mathrm{My}-\mathrm{La}$ and $\mathrm{HaCaT}$ (unstimulated or stimulated) cells according to previously described protocols [17, 20]. Fifty million crosslinked cells were lysed and the chromatin digested with HindIII at $37^{\circ} \mathrm{C}$ overnight. Restriction cut sites were filled in using dCTP, dGTP, dTTP and biotin-14-dATP (Life Technologies), then innucleus ligation was carried out at $16^{\circ} \mathrm{C}$ for $4-6 \mathrm{~h}$. Crosslinks were removed by proteinase- $\mathrm{K}$ overnight at $65^{\circ} \mathrm{C}$, and RNA was digested using RNaseA for $60 \mathrm{~min}$ at $37^{\circ} \mathrm{C}$. The DNA was purified by sequential phenol and phenol-chloroform extractions and ethanolprecipitated at $-20^{\circ} \mathrm{C}$ overnight, followed by two further phenol-chloroform extractions and a second overnight precipitation.

A 40- $\mu \mathrm{g}$ aliquot of DNA was taken forward for further processing following QC steps. T4 DNA polymerase was used to remove biotin-14-dATP from non-ligated ends then the DNA purified by phenol-chloroform extraction and ethanol precipitation overnight. The DNA was sheared using a Covaris S220 sonicator, and end-repair was performed using T4 DNA polymerase, T4 DNA polynucleotide kinase and DNA polymerase I, large (Klenow) fragment. The sample was purified using Qiagen MinElute Kit, with a modified protocol described by [55]. Klenow (exo-) was used to adenylate DNA fragment ends, and a double-sided SPRI bead size selection was used to obtain fragments of approximately 200-600 bp. Dynabeads MyOne Streptavidin C1 beads (Life Technologies) were used to pull down biotinylated fragments, which were then ligated to annealed Illumina sequencing adapters. PCR was performed using Phusion HF (NEB) and TruPE PCR primers (Illumina), then the amplified DNA was cleaned twice using $1.8 \times$ volume of SPRI beads.

Amplified DNA up to $750 \mathrm{ng}$ was concentrated using a vacuum concentrator and bound to the capture baits in a single hybridisation reaction using SureSelectXT reagents and protocol by Agilent Technologies. The biotinylated baits were captured using Dynabeads MyOne 
Streptavidin T1 beads (Life Technologies). Following washes, the libraries were amplified on the beads using Phusion HF and barcoded TruPE primers then the amplified DNA cleaned twice using $1.8 \times$ volume of SPRI beads. The quality and quantity of the Capture $\mathrm{Hi}-\mathrm{C}$ libraries were tested by Bioanalyzer and KAPA qPCR (Kapa Biosystems). Capture Hi-C libraries were analysed by 75-bp paired-end next-generation sequencing on an Illumina NextSeq500 at the Genomic Technologies Core Facility at the University of Manchester (My-La) or HiSeq 4000 at Edinburgh Genomics at the University of Edinburgh ( $\mathrm{HaCaT})$.

$\mathrm{CHi}-\mathrm{C}$ sequence data were processed through the HiC User Pipeline (HiCUP) v0.5.8 [56]. For each cell type, the two biological replicates were simultaneously run through CHiCAGO v1.1.8 [57] in R v3.3.0, and significant interactions were called with a score threshold of 5 . To down-sample My-La libraries in order to more closely mirror the number of $\mathrm{HaCaT}$ on-target di-tags, we used DownsampleSam from Picard v2.9.2 [58] with the option $P=0.3$ (replicate 1 ) and $P=0.4$ (replicate 2), followed by HiCUP and CHiCAGO pipelines as above. Reproducibility was assessed firstly by observing the number of shared interactions between replicates. We generated Venn diagrams displaying the shared interactions (CHiCAGO score $\geq 5$ ) between each replicate called individually, or both replicates combined in the CHiCAGO function. We used HiCRep [25] to assess the correlation between samples by firstly filtering for reads with a captured bait at one or both ends, using the shared baits across samples, and then creating 10-kb raw matrices on chromosome 1 using HOMER [59]. Matrices were submitted to HiCrep in all pairwise combinations with the following parameters: resolution $=10 \mathrm{~kb}$, smoothing parameter $(h)=3$ and maximum distance $=5 \mathrm{Mb}$.

To detect enrichment of features in the $\mathrm{CHi}-\mathrm{C}$ interactions, we first obtained narrowPeak bed files of H3K4me3, H3K27ac and CTCF as follows: H3K27ac in NHEK (ENCODE; ENCFF943CBQ), H3K4me3 in NHEK (ENCODE; ENCFF027FJC), H3K27ac in primary CD8+ naive $\mathrm{T}$ cells (Roadmap Epigenomics; E047), H3K4me3 in primary CD8+ naive $\mathrm{T}$ cells (Roadmap Epigenomics; E047) and CTCF in NHEK (ENCODE; ENCFF226MQR). We obtained transcription start sites (Ensembl 99) for all genes expressed in the corresponding cell type in our RNA-seq data using Ensembl BioMart. The "peakEnrichment4Features" function in CHiCAGO was used to detect enrichment of each feature in the $\mathrm{CHi}-\mathrm{C}$ data.

To determine the differences in the interaction distances, we restricted the data to psoriasis loci and obtained all distances in cis for each cell line. We then performed a Kruskal-Wallis test using Dunn's multiple comparisons to determine cell-specific differences. BEDTools v2.17.0 [60] was used to detect interactions between psoriasis- associated fragments and gene promoters, defined by fragments covering regions within $500 \mathrm{bp}$ of transcription start sites (Ensembl release 75; GRCh37). To validate our CHi-C data, we overlapped the interactions with psoriasis GWAS SNPs colocalised with eQTLs [27]. Sixteen out of 26 of their reported proxies overlapped HindIII fragments in our $\mathrm{CHi}-\mathrm{C}$ data; the lack of complete overlap mostly owed to our study prioritising index SNPs from more recent metaanalyses over older studies. In addition, we were not able to design baits for one of the reported regions at rs7552167, as described above. We then determined how many of the target genes were implicated by $\mathrm{CHi}-\mathrm{C}$ interactions, as visualised on the WashU Epigenome Browser [31]. We collated a list of all psoriasis GWAS SNPs or proxies that fell within baited fragments interacting with expressed genes $(N=$ 1776) and determined if they altered known transcription factor binding motifs using the tool SNP2TFBS [29].

\section{$\mathrm{Hi}-\mathrm{C}$}

For each cell type, a single $\mathrm{Hi}-\mathrm{C}$ library was generated by re-amplifying the pre-Capture Hi-C library bound to streptavidin beads, using Phusion HF and barcoded TruPE primers. The amplified DNA was cleaned twice using $1.8 \times$ volume of SPRI beads. The quality and quantity of the Hi-C libraries were tested by Bioanalyzer and KAPA qPCR. Hi-C libraries were analysed by nextgeneration sequencing. The My-La Hi-C library was sequenced on an Illumina HiSeq 2500 generating 100-bp paired ends at the Babraham Institute Sequencing Facility, Cambridge. The $\mathrm{HaCaT} \mathrm{Hi}-\mathrm{C}$ libraries were sequenced on an Illumina HiSeq 4000 generating 75-bp paired ends at Edinburgh Genomics at the University of Edinburgh. The sequence data was filtered, and the adapters were removed using fastp v0.19.4 [61]. The reads were then mapped to the GRCh38 genome with $\mathrm{Hi}-\mathrm{C}$ Pro v2.11.0 [62], using default settings. The Hi-C interaction matrices were normalised within $\mathrm{Hi}-\mathrm{C}$ Pro using iterative correction and eigenvector decomposition (ICE). Topologically associating domains (TADs) were then called in the TADtool software [63] using insulation score with the normalised Hi-C contact matrices, binned with $40 \mathrm{~kb}$ resolution. TADs were visualised alongside $\mathrm{CHi}-\mathrm{C}$ interactions on the WashU Epigenome Browser [31].

\section{HiChIP}

For each cell type, HiChIP libraries were generated according to the Chang Lab protocol [21] in biological duplicate. Ten million crosslinked cells were lysed and the chromatin digested using $375 \mathrm{U}$ of MboI (NEB, R0147M) for $4 \mathrm{~h}$ at $37^{\circ} \mathrm{C}$. Fragment ends were filled in using dCTP, dGTP, dTTP and biotin-14 dATP (Life Technologies) and ligated at room temperature overnight. The nuclei were lysed and the chromatin sheared to lengths of approximately $200-700$ bp using a Covaris 
S220. Immunoprecipitation was performed overnight at $4{ }^{\circ} \mathrm{C}$ using $20 \mu \mathrm{g}$ of H3K27ac antibody (Abcam ab4729). The DNA was captured on a 1:1 mixture of protein A and G Dynabeads (Invitrogen 10001D and 10003D). After washes, the DNA was eluted with proteinase $\mathrm{K}$ at $65^{\circ} \mathrm{C}$ overnight. The sample was cleaned using Zymo Clean and Concentrator Columns (Zymo D4013) and quantified using the Qubit DNA HS kit. Twenty to $35 \mathrm{ng}$ of DNA was taken forward for biotin pulldown with streptavidin $\mathrm{C}-1$ beads at room temperature for $30 \mathrm{~min}$. The beads were suspended in TD buffer from the Nextera kit and transposed with $\mathrm{Tn} 5$ (Illumina) at $55^{\circ} \mathrm{C}$ for exactly $10 \mathrm{~min}$. The volume of Tn5 was dependent on DNA quantity and defined by the original HiChIP protocol [21]. After washes, the library was amplified off the beads using Phusion polymerase and Nextera indexing primers (Illumina). SPRI beads were used to select fragments approximately $300-700 \mathrm{bp}$ in length. Quantification and quality control of the final HiChIP library was conducted using a Bioanalyzer and KAPA quantification kit (Kapa Biosystems). Libraries underwent next-generation sequencing on a HiSeq 2500 at the Babraham Institute Sequencing Facility, Cambridge, generating 100-bp paired ends.

Sequencing data for the HiChIP libraries was filtered, and the adapters were removed using fastp v0.19.4. The reads were then mapped to the GRCh38 genome with HiC Pro v2.11.0, using default settings. The two replicates were merged, and loops were identified using FitHiChIP [64] using the following settings: coverage normalisation, stringent background with merging enabled, peaks generated from HiChIP data using the included tool and $5-\mathrm{kb}$ bin size. The interactions were filtered to those originating from the H3K27ac peak on the KLF4 promoter, or the psoriasis SNPs, before being uploaded for visualisation on the WashU Epigenome Browser [31].

To compare H3K27ac signal in shared peaks between unstimulated and stimulated $\mathrm{HaCaT}$ cells in 9q31.2, genome-wide anchors were called in hichipper v0.7.3 [65] using the self-circle and dangling end reads for the first replicate, extended for $147 \mathrm{bp}$. These peaks were first combined to produce a merged peak set, and then the signal from the two conditions was intersected on the peaks using BEDTools map function and the mean signal for each peak was reported for each condition. The resulting values were imported in $\mathrm{R}$ and normalised using DESeq2 estimate size factors function [66]. The normalised counts for peaks within the 9q31.2 TAD (chr9:110202281-111602280) were compared between the two conditions using a Wilcoxon matched-pairs signed-rank test in GraphPad Prism.

\section{RNA-seq}

3' mRNA sequencing libraries were generated for cell lines using the QuantSeq 3' mRNA-Seq Library Prep Kit
FWD for Illumina (Lexogen). RNA-seq libraries were generated for unstimulated $\mathrm{HaCaT}$ cells $(N=4)$, stimulated HaCat cells $(N=3)$ and My-La cells $(N=1)$. Libraries were sequenced using single-end Illumina SBS technology. Reads were quality trimmed using Trimmomatic v0.38 [67] using a sliding window of 5 with a mean minimum quality of 20 . Adapters and poly A/poly $\mathrm{G}$ tails were removed using Cutadapt v1.18 [68], and then UMIs were extracted from the $5^{\prime}$ of the reads using UMI-tools v0.5.5 [69]. Reads were then mapped using STAR v2.5.3a [70] on the GRCh38 genome with GENCODE annotation v29. Reads were de-duplicated using UMIs with UMI-tools and then counted using HTSeq v0.11.2 [71]. Count matrixes were analysed in $\mathrm{R}$ 3.5.1, and normalisation and differential expression analysis was conducted using DESeq2 v1.22.2. Differentially expressed genes were called with an adjusted $P$ value of 0.10 (FDR 10\%). Gene set enrichment pathway analysis was performed using GAGE v2.32.1 [72] using "normal" shrinked log fold changes from DESeq2. For the detection of expressed genes in the cell lines, we considered RNA-seq counts greater than 1 in at least one of the sequenced samples.

\section{Functional annotation in $9 q 31.2$}

SNPs in LD $\left(r^{2}>0.8\right)$ with the lead SNP rs10979182 were examined for their intersection with ENCODE data for histone marks, transcription factor binding sites and DNase hypersensitivity. RegulomeDB v1.1 [73] was used to rank the SNPs based on the likely regulatory function. The SNPs were also assessed using Haploreg v4.1 [74], which includes expression quantitative trait loci (eQTL) data from several studies including GTEx [75] and GEUVADIS [76].

\section{C-qPCR in 9q31.2}

$3 \mathrm{C}$ libraries were generated in biological triplicate as previously described [77]. Twenty to 30 million crosslinked cells were lysed, digested, ligated and purified as described in the first section of the Capture $\mathrm{Hi}-\mathrm{C}$ protocol above, omitting the biotinylation step. Control libraries were constructed using bacterial artificial chromosome (BAC) clones as described by [77]. A set of 11 minimally overlapping BAC sequences was compiled to span the locus (chr9:110168556-111889073, hg19): RP11-795I4, CTD-2258 L2, RP11-762G1, RP11-358A7, RP11-779 J13, CTD-2517A7, RP11-454G15, RP11585H18, CTD-2333H8, CTD-2649 N21 and RP11$316 \mathrm{H} 9$. BAC clone identity was confirmed by PCR to amplify selected regions at either end of the sequence. BAC DNA was combined in equimolar quantities to a total of $20 \mu \mathrm{g}$ and digested with HindIII overnight at $37^{\circ} \mathrm{C}$. The DNA was purified with phenol-chloroform and precipitated in ethanol for several hours at $-20^{\circ} \mathrm{C}$. 
Ligation was performed at $16^{\circ} \mathrm{C}$ overnight using $\mathrm{T} 4$ DNA ligase. Two further phenol-chloroform extractions were performed followed by a chloroform extraction, and the DNA was precipitated in ethanol for several hours at $-20^{\circ} \mathrm{C}$. $3 \mathrm{C}$ libraries and $\mathrm{BAC}$ control libraries were quantified using a Qubit dsDNA BR kit.

qPCR was carried out using SYBR Green or TaqMan technology to determine the interaction frequencies in the 9q31.2 locus. Unidirectional primers were designed using Primer3 (http://primer3.ut.ee/) [78] to complement the sequences approximately $50-100 \mathrm{bp}$ from the target HindIII cut site (primers shown in Additional file 13 Table S18). For the TaqMan experiment, an additional TaqMan probe was designed to bind to a region between the anchor primer and the restriction cut site (probe and primers shown in Additional file 13 Table S19). For each anchor fragment, a primer was designed to target a short-range control region located 2-3 fragments further along the sequence. qPCRs were carried out in technical triplicate, and 10-fold dilutions of the BAC template (50-0.005 ng) were included alongside the $3 \mathrm{C}$ library templates for each tested interaction. For each primer pair, BAC curves were generated from $\log _{10}$ of the library concentration against the average $\mathrm{Ct}$ value across PCR triplicates. The relative interaction frequencies were calculated in the following manner:

$$
\text { Interaction frequency }(F)=10^{((\mathrm{Ct}-i) / s)}
$$

where $\mathrm{Ct}$ is the measured cycle threshold value of $3 \mathrm{C}$ library (mean of PCR triplicates), $i$ is the $Y$ intercept of BAC curve and $s$ is the slope of BAC curve.

The interaction frequencies were then normalised to the short-range control as follows:

$$
\text { Relative interaction frequency }(R)=\frac{F[\text { short-range control }]}{F[\text { test interaction }]}
$$

where $F$ is the interaction frequency.

Significant interactions were detected using one-way ANOVA in GraphPad Prism 7 with Dunnett's or Tukey's test for multiple comparisons dependent on whether there was a single negative control region or each interaction was compared to all other interactions, respectively.

\section{ChIP-qPCR in 9q31.2}

Chromatin immunoprecipitation (ChIP) libraries were generated as previously described [49]. Ten million crosslinked cells were lysed, and the chromatin was fragmented to optimal lengths of 200-400 bp using a Covaris S220. A volume of chromatin corresponding to approximately 1 million cells was immunoprecipitated with a rabbit polyclonal antibody for H3K4me1 (Abcam ab8895) or H3K27ac (ab4729) or a negative control rabbit IgG (Diagenode C15410206) with a mixture of Dynabeads $\mathrm{A}$ and $\mathrm{G}$ at $4{ }^{\circ} \mathrm{C}$ overnight. After washes, the DNA was eluted with proteinase $\mathrm{K}$ at $62^{\circ} \mathrm{C}$ for $2 \mathrm{~h}$ and then $95^{\circ} \mathrm{C}$ for $10 \mathrm{~min}$. The DNA was purified using Purelink Quick PCR purification columns (Life Technologies). ChIP enrichment was measured at loci of interest by qPCR using SYBR Green or TaqMan technology. The data were normalised by calculating the percentage of total chromatin that was immunoprecipitated in comparison with an input sample. Negative controls included no-antibody and IgG-precipitated samples.

For SYBR experiments, primers were designed using Primer3 (http://primer3.ut.ee/) to target regions of 100$200 \mathrm{bp}$ encompassing likely regulatory SNPs or enhancers in 9q31.2 (for primers, see Additional file 13, Table S20). For the allele-specific analysis at rs6477612, a TaqMan SNP genotyping probe was acquired that detected C (risk) or $\mathrm{T}$ (protective) alleles (Applied Biosystems, assay ID C_29343482_10). The difference in antibody binding to each allele was determined by the percentage of ChIP enrichment in comparison with the signal for each allele obtained from the input sample. ChIP-qPCR data were analysed in GraphPad Prism using two-way ANOVA. Experiments were performed in biological triplicate ( $\mathrm{HaCaT}$ and $\mathrm{My}$-La lines) or duplicate (NHEK cells).

\section{CRISPR activation in 9q31.2}

CRISPR activation using the catalytically inactive Cas9 (dCas9)-P300 complex was performed in $\mathrm{HaCaT}$ cells to determine the role of the four putative enhancers in 9q31.2. Firstly, a HaCaT cell line stably expressing dCas9P300 was generated using the CRISPRa plasmid pLV dCas9-p300-P2A-PuroR plasmid (Addgene 83889) [79]. Briefly, plasmid DNA was combined with third-generation viral packaging components and polyethyenimine (PEI) in the ratio 1:6 (total DNA:PEI). The DNA:PEI complexes were added to Lenti-X 293T cells, which were then incubated for $72 \mathrm{~h}$, after which the lentivirus-containing supernatant was harvested and filtered to remove cell debris. Lentiviral transduction of 300,000 HaCaT cells was carried out using $1 \mathrm{~mL}$ of the unconcentrated lentivirus and $8 \mu \mathrm{g} /$ $\mathrm{mL}$ polybrene. The cells were grown for several days before selection with $1 \mu \mathrm{g} / \mathrm{mL}$ puromycin for 7 days, after which the $\mathrm{HaCaT}$ dCas9-P300 cell population was maintained with $0.5 \mu \mathrm{g} / \mathrm{mL}$ puromycin.

To select sgRNA in 9q31.2, SNPs in $r^{2}>0.8$ with rs10979182 were prioritised by their overlap with enhancer elements, defined by active regulatory regions in NHEK according to ChromHMM [11]. sgRNA sequences were designed using the online CRISPOR tool [80] to target loci within $200 \mathrm{bp}$ of the prioritised SNPs $($ mean $=85 \mathrm{bp})$. sgRNA were selected based on specificity score and proximity to the SNP. In total, there were 
27 SNPs; two of these could not be targeted by sgRNA within 200 bp (rs7019552 and rs11355519), and another two SNPs, rs4979624 and rs7029094, were captured by a single sgRNA targeting the intervening region. In total, therefore, there were $24 \mathrm{sgRNA}$; these were grouped into four pools of 5-7 SNPs to target the four putative enhancers (Additional file 13, Table S21 and Additional file 11, Fig. S8).

For each of the sgRNA pools and controls (scrambled guide or empty plasmid), we generated three biological replicate cell lines as follows. The sgRNA sequences were cloned into the pLKO5.sgRNA.EFS.GFP plasmid (Addgene 57,822) [81], and equimolar plasmid pools were generated for each enhancer. The plasmid pools were then packaged using the same lentiviral method as the dCas9-P300 plasmid. The guide pools were introduced into the stable $\mathrm{HaCaT}$ dCas9-P300 cells using a second round of lentiviral transduction, and cells that had integrated the sgRNA plasmids were isolated by flow cytometry for GFP. RNA was extracted from the sorted cells using the RNeasy mini kit (Qiagen). qPCR was performed to assay gene expression using the TaqMan RNA-to-Ct 1step kit (Thermo Fisher Scientific) using the following TaqMan assays for genes in the 9q31 locus: $R A D 23 B$ (Hs00234102_m1), KLF4 (Hs00358836_m1), ACTL7A (Hs00246418_s1), ACTL7B (Hs00246411_s1), IKBKAP (Hs00175353_m1), FAM206A (Hs00607423_m1) and CTNNAL1 (Hs00972098_m1). Delta-delta Ct analysis was conducted against a control HaCaT dCas9-P300 cell line transduced with the sgRNA plasmid containing a previously published scrambled, non-targeting insert (Scramble2 [82];). Two housekeeping gene assays, TBP (Hs00427620_m1) and YWHAZ (Hs01122445_g1), were used for normalisation. For statistical analysis, we performed a Kruskal-Wallis test of the fold changes and used Dunn's multiple comparisons test to determine the differences between cells with the scrambled guide or with the pools of sgRNA.

For the CRISPRa pool with the greatest impact on KLF4 expression, RNA-seq and gene set enrichment analyses were performed as described above. In addition, differentially expressed genes were processed through the STRING database to identify potential proteinprotein interaction networks [83]. Next, we used Lisa to search for transcriptional regulators of the differentially expressed gene set [48]. The genes were uploaded to the Lisa portal, and the combined model, based on CistromeDB TR ChIP-seq, was retrieved.

In addition to the above, the validity of the $\mathrm{HaCaT}$ dCas9-P300 system was first tested using sgRNA directed to the promoters of ILIRN or SLC4A1; these sgRNA have previously been shown to be effective in CRISPRa (IL1RN: sgRNA CR3 [84]; SLC4A1: Weissman Lab). The upregulation of these genes was detected by
TaqMan qPCR for IL1RN (Hs00893626_m1) and SLC4A1 (Hs00978607_g1) (Additional file 14, Fig. S9).

\section{Supplementary information}

Supplementary information accompanies this paper at https://doi.org/10. 1186/s12915-020-00779-3.

\section{Additional file 1 : Table S1. Psoriasis SNPS included in the capture} design. Table S2. $\mathrm{CHi}-\mathrm{C}$ library metrics.

Additional file $\mathbf{2}$ : Figure S1. Reproducibility between $\mathrm{CHi}-\mathrm{C}$ replicates for $\mathrm{HaCaT}$ (unstimulated), HaCaT (stimulated with IFN- $\gamma$ ), and My-La (down-sampled). A) The number of shared cis-interactions (CHiCAGO score $\geq 5$ ) between replicates are shown in a scaled Venn diagram for each cell type. Each $\mathrm{CHi}-\mathrm{C}$ replicate was individually analysed by $\mathrm{CHi}-$ CAGO, as well in the combined analysis where both replicates were submitted to $\mathrm{CHiCAGO}$ together. B) Correlation between $\mathrm{CHi}-\mathrm{C}$ samples was assessed using HiCRep [25] for $10 \mathrm{~kb}$ interaction bins on chromosome 1. The heatmap shows the reported stratum-adjusted correlation coefficient (SCC) between samples.

Additional file 3 : Figure S2. Enrichment of features within other-ends of $\mathrm{CHi}-\mathrm{C}$ interactions. The peak locations of H3K4me3, H3K27ac and CTCF in NHEK (ENCODE), H3K4me3 and H3K27ac in primary CD8+ naïve T cells (Roadmap Epigenomics) [85] and transcription start sites (Ensembl 99) of active genes (read counts $>0$ ) according to the RNA-seq data in HaCaT and My-La cell lines in the present study were tested against other-ends of interactions with all targeted autoimmune loci using the peakEnrichment4Features function of the CHiCAGO package [57]. The graphs show the number of overlaps with the feature in the interaction data (yellow) versus the mean number of overlaps in 100 sampled interactions from the non-significant pool (blue). Error bars show the 95\% confidence interval.

Additional file 4 : Figure S3. Frequency distributions of distances between psoriasis bait fragments and interacting fragments in the $\mathrm{CHi}-\mathrm{C}$ experiment. The frequency of interactions is shown for $50 \mathrm{~kb}$ bins up to $3 \mathrm{Mb}$ in $\mathrm{HaCaT}$ unstimulated (A), HaCaT stimulated (B) and My-La cells (C).

Additional file $\mathbf{5}$ : Table S3. Validation analysis of known eQTLs within the $\mathrm{CHi}-\mathrm{C}$ data. Tables S4-6. CHi-C interactions between psoriasis loci and gene promoters with associated expression data. For each locus, the top interaction is shown between the psoriasis bait fragment and the gene promoter fragment in $\mathrm{HaCaT}$ unstimulated (S4), stimulated (S5) and My-La (S6).

Additional file $\mathbf{6}$ : Figure S4. Frequency distributions of the number of interactions with promoter fragments per psoriasis-associated bait fragment in the $\mathrm{CHi}-\mathrm{C}$ experiment. To determine the frequency distribution of psoriasis bait-promoter interactions, the data was firstly restricted to interactions between psoriasis-associated bait fragments and promoter fragments ("Promoter Interactions"). Next, the number of promoter fragments per bait fragment was counted. Of those promoter fragments, the number of corresponding gene promoters was determined. This was necessary because some gene promoters share the same fragment, and some gene promoters are found in more than one fragment. The number of interacting promoter fragments per bait fragment in Promoter Interactions are shown for HaCaT unstimulated (A), HaCaT stimulated (C) and My-La (E). The number of corresponding gene promoters are shown for HaCaT unstimulated (B), HaCaT stimulated (D) and My-La (F). The interaction frequencies are shown in bins of 1 .

Additional file 7 : Table S7. RNA-seq data: all normalised counts across the three cell lines. Table S8. Lists of expressed genes intersecting psoriasis bait fragments. Table S9. Enrichment of TFBSs among psoriasis GWAS SNPs interacting with promoters of active genes, using SNP2TFBS tool. Table S10. Differentially expressed genes between unstimulated and stimulated (IFNg) HaCaT cells. Table S11. GO term enrichments for DE genes in stimulation experiment. Table S12. DE genes interacting with psoriasis baits in unstimulated and stimulated HaCaT cells.

Additional file 8 : Figure S5. 3C-qPCR results in the $9 q 31.2$ locus anchored at the Hindlll fragment containing the third psoriasis-associated 
putative enhancer (rs6477612). qPCR was carried out on HaCaT and MyLa $3 C$ libraries using SYBR ${ }^{\bullet}$ Green as the reporter. The anchor fragment at the third psoriasis-associated enhancer is at distance $0 \mathrm{~kb}$. Test fragments were selected in and around KLF4, two points in the gene desert and at fragments containing gene promoters for IKBKAP, FAM206A and CTNNAL1. Interactions were normalised to a short range control. Asterisks denote fragments that had a significantly higher relative interaction frequency than one or more of the other tested fragments, after multiple testing (one-way ANOVA, adjusted $P$-value $<0.05$ ). Bars show mean + SD of triplicate $3 C$ libraries. Abbreviations: Cent, centromeric; Int, intergenic.

Additional file 9 : Figure S6. 3C-qPCR results in the 9q31.2 locus from the HindIII fragment containing the KLF4 gene and promoter. GPCR was carried out on $\mathrm{HaCaT}$ and My-La $3 \mathrm{C}$ libraries using TagMan ${ }^{\circledR}$ as the reporter. The anchor fragment (distance 0) contained the entire KLF4 gene and promoter. An intergenic fragment located approximately $200 \mathrm{~kb}$ from the anchor fragment was utilised as a negative control region. Eleven test fragments were selected at regular intervals across the psoriasis association. The positive controls in the Dryden BrCa region were included. Asterisks denote fragments that had a significantly higher relative interaction frequency than the NCR (one-way ANOVA, adjusted P-value < 0.05). Bars show mean + SD of triplicate $3 C$ libraries. Abbreviations: Int, intergenic; $\mathrm{NCR}$, negative control region; $\mathrm{BrCa}$, breast cancer.

Additional file 10 : Figure S7. Previously reported HiC interaction data in NHEK cells in the 9q31.2 locus [14]. Interactions are indicated between KLF4 and the gene desert, including the psoriasis SNPs and the breast cancer region shown in a previous $\mathrm{CHi}-\mathrm{C}$ experiment [17]. Image created using the YUE lab 3D Genome Browser.

Additional file 11 : Figure S8. sgRNA pools targeting the four putative enhancers in the psoriasis susceptibility locus at 9q31.2. a) location on chromosome 9; b) sgRNA locations; c) SNPs in LD with rs10979182 overlapping putative enhancers; d) ChromHMM segments in NHEK where red, yellow and green indicate "active TSS", "enhancers" and "transcription" respectively; e) H3K4me1 (ENCODE); f) H3K27ac (ENCODE); g) DNase clusters (ENCODE); h) transcription factor ChIP (ENCODE).

Additional file 12 : Table S13. Differentially expressed genes between $\mathrm{HaCaT}$ cells containing dCas9 P300 with scrambled guide or 9q31.2 Pool 3 guide in CRISPRa experiment. Table S14. GO term enrichments for DE genes in CRISPRa experiment. Table S15. STRING results for DE genes in CRISPRa experiments. Table S16. TF binding sites in DE genes (LISA result). Table S17. Annotated variants in LD with rs10979182 in 9q31.2.

Additional file 13 : Table S18. Primers used in the first $9 q 31.23 C$ (SYBR) assay. Table S19. Primers used in the second 9q31.2 3C (TaqMan) assay. Table S20. Primers used in ChIP experiments. Table S21. sgRNA used in the CRISPRa experiment.

Additional file $\mathbf{1 4}$ : Figure S9. Effect of sgRNA targeting ILIRN and SLC4A1 promoters in HaCaT dCas9 P300 cells. HaCaT cells expressing dCas9 P300 were transduced with sgRNA plasmids containing previouslypublished sgRNA for the ILIRN promoter or the SLC4A1 promoter, in biological triplicate. Control cell lines were generated by transducing $\mathrm{HaCaT}$ dCas9 P300 cells with a sgRNA plasmid containing a scrambled guide (Scr) or no guide insert (Plasmid only; PO). aPCR was carried out using TaqMan assays for ILIRN or SLC4A1. Housekeeping genes used were TBP and YWHAZ. Graphs show fold change of gene expression relative to the cells containing the scrambled sgRNA. One-way ANOVA was carried out in GraphPad Prism: in both cases, the cells containing the targeting sgRNA had significantly higher gene expression than the scrambled control $(P=0.002$ for $I L 1 R N ; P=0.0001$ for $S L C 4 A 1)$. Asterisks denote $P<0.05$. Graphs show the mean fold-change in comparison with scrambled guide, +- SEM of triplicate cell lines.

\section{Acknowledgements}

The authors would like to acknowledge the anonymous reviewers for their helpful and constructive comments. The authors also wish to acknowledge the assistance given by IT Services and the use of the Computational Shared Facility at The University of Manchester.

\section{Authors' contributions}

All authors contributed to the preparation of the manuscript. SE, RBW, KD and HRJ contributed to the conception and design of the experiment. HRJ, $\mathrm{KD}, \mathrm{AMG}, \mathrm{PM}, \mathrm{CS}, J \mathrm{H}, \mathrm{OG}, \mathrm{CT}, \mathrm{JD}, \mathrm{VPG}, \mathrm{YF}$ and $\mathrm{PG}$ contributed to the acquisition or analysis of the data. HRJ, SE, RBW, KD, CS, AY, APM, AA, PM and $\mathrm{GO}$ contributed to the interpretation of the results. All authors read and approved the final manuscript.

\section{Funding}

This work was co-funded by the NIHR Manchester Biomedical Research Centre, Versus Arthritis (grant refs. 21348 and 21754) and a PhD studentship awarded to HRJ by The Sir Jules Thorne Charitable Trust.

\section{Availability of data and materials}

The sequence datasets generated and analysed during the current study are available in the GEO repository under accession number GSE137906.

\section{Ethics approval and consent to participate}

Not applicable

\section{Consent for publication}

Not applicable

\section{Competing interests}

The authors declare no competing interests.

\section{Author details}

${ }^{1}$ Centre for Genetics and Genomics Versus Arthritis, Division of Musculoskeletal and Dermatological Sciences, School of Biological Sciences, Faculty of Biology, Medicine and Health, The University of Manchester, Manchester, UK. ${ }^{2}$ Dermatology Centre, Manchester NIHR Biomedical Research Centre, Manchester Academic Health Science Centre, Salford Royal NHS Foundation Trust, Manchester, UK. ${ }^{3}$ The Lydia Becker Institute of Immunology and Inflammation, The University of Manchester, Manchester, UK. ${ }^{4}$ Genome Editing Unit, Faculty of Biology, Medicine and Health, The University of Manchester, Manchester, UK. ${ }^{5}$ Genes and Human Disease Research Program, Oklahoma Medical Research Foundation, Oklahoma City, OK 73104, USA.

Received: 5 November 2019 Accepted: 14 April 2020

Published online: 04 May 2020

\section{References}

1. Barker J, Mitra RS, Griffiths CEM, Dixit VM, Nickoloff BJ. Keratinocytes as initiators of inflammation. Lancet. 1991;337(8735):211-4.

2. Schon MP, Boehncke WH. Medical progress - psoriasis. N Engl J Med. 2005; 352(18):1899-912.

3. Schlaak JF, Buslau M, Jochum W, Hermann E, Girndt M, Gallati H, et al. Tcells involved in psoriasis-vulgaris belong to the Th1 subset. J Investig Dermatol. 1994;102(2):145-9.

4. Lowes MA, Bowcock AM, Krueger JG. Pathogenesis and therapy of psoriasis. Nature. 2007;445(7130):866-73.

5. Hijnen D, Knol EF, Gent YY, Giovannone B, Beijn SJ, Kupper TS, et al. CD8(+) $T$ cells in the lesional skin of atopic dermatitis and psoriasis patients are an important source of IFN-gamma, IL-13, IL-17, and IL-22. J Invest Dermatol. 2013;133(4):973-9.

6. Albanesi C, Madonna S, Gisondi P, Girolomoni G. The interplay between keratinocytes and immune cells in the pathogenesis of psoriasis. Front Immunol. 2018:9:1549.

7. Tsoi LC, Stuart PE, Tian C, Gudjonsson JE, Das S, Zawistowski M, et al. Large scale meta-analysis characterizes genetic architecture for common psoriasis associated variants. Nat Commun. 2017:8:8.

8. Yin X, Low HQ, Wang L, Li Y, Ellinghaus E, Han J, et al. Genome-wide metaanalysis identifies multiple novel associations and ethnic heterogeneity of psoriasis susceptibility. Nat Commun. 2015;6:6916.

9. Zuo X, Sun L, Yin X, Gao J, Sheng Y, Xu J, et al. Whole-exome SNP array identifies 15 new susceptibility loci for psoriasis. Nat Commun. 2015;6:6793.

10. Kundaje A, Meuleman W, Ernst J, Bilenky M, Yen A, Heravi-Moussavi A, et al. Integrative analysis of 111 reference human epigenomes. Nature. 2015;518: 317. 
11. Ernst J, Kheradpour P, Mikkelsen TS, Shoresh N, Ward LD, Epstein CB, et al. Mapping and analysis of chromatin state dynamics in nine human cell types. Nature. 2011;473(7345):43-9.

12. Farh KK, Marson A, Zhu J, Kleinewietfeld M, Housley WJ, Beik S, et al. Genetic and epigenetic fine mapping of causal autoimmune disease variants. Nature. 2015;518(7539):337-43.

13. Javierre BM, Burren OS, Wilder SP, Kreuzhuber R, Hill SM, Sewitz $S$, et al. Lineage-specific genome architecture links enhancers and non-coding disease variants to target gene promoters. Cell. 2016;167(5):1369-84 e19.

14. Rao SS, Huntley MH, Durand NC, Stamenova EK, Bochkov ID, Robinson JT, et al. A 3D map of the human genome at kilobase resolution reveals principles of chromatin looping. Cell. 2014;159(7):1665-80.

15. Ray-Jones H, Eyre S, Barton A, Warren RB. One SNP at a time: moving beyond GWAS in psoriasis. J Invest Dermatol. 2016;136(3):567-73.

16. Lieberman-Aiden E, van Berkum NL, Williams L, Imakaev M, Ragoczy T, Telling $A$, et al. Comprehensive mapping of long-range interactions reveals folding principles of the human genome. Science. 2009;326(5950):289-93.

17. Dryden NH, Broome LR, Dudbridge F, Johnson N, Orr N, Schoenfelder S, et al. Unbiased analysis of potential targets of breast cancer susceptibility loci by Capture Hi-C. Genome Res. 2014;24(11):1854-68.

18. Baxter JS, Leavy OC, Dryden NH, Maguire S, Johnson N, Fedele V, et al. Capture Hi-C identifies putative target genes at 33 breast cancer risk loci. Nat Commun. 2018;9(1):1028.

19. Jager R, Migliorini G, Henrion M, Kandaswamy R, Speedy HE, Heindl A, et al. Capture $\mathrm{Hi}-\mathrm{C}$ identifies the chromatin interactome of colorectal cancer risk loci. Nat Commun. 2015;6:6178.

20. Martin P, McGovern A, Orozco G, Duffus K, Yarwood A, Schoenfelder S, et al. Capture $\mathrm{Hi}-\mathrm{C}$ reveals novel candidate genes and complex long-range interactions with related autoimmune risk loci. Nat Commun. 2015;6:10069.

21. Mumbach MR, Rubin AJ, Flynn RA, Dai C, Khavari PA, Greenleaf WJ, et al. HiChIP: efficient and sensitive analysis of protein-directed genome architecture. Nat Methods. 2016;13(11):919-22.

22. Mumbach MR, Satpathy AT, Boyle EA, Dai C, Gowen BG, Cho SW, et al. Enhancer connectome in primary human cells identifies target genes of disease-associated DNA elements. Nat Genet. 2017;49(11):1602-12.

23. Pelikan RC, Kelly JA, Fu Y, Lareau CA, Tessneer KL, Wiley GB, et al. Enhancer histone-QTLs are enriched on autoimmune risk haplotypes and influence gene expression within chromatin networks. Nat Commun. 2018;9:14.

24. Adli M. The CRISPR tool kit for genome editing and beyond. Nat Commun. 2018:9(1):1911.

25. Yang T, Zhang F, Yardımcı GG, Song F, Hardison RC, Noble WS, et al. HiCRep: assessing the reproducibility of $\mathrm{Hi}-\mathrm{C}$ data using a stratum-adjusted correlation coefficient. Genome Res. 2017;27(11):1939-49.

26. Netchiporouk E, Gantchev J, Tsang M, Thibault P, Watters AK, Hughes JM, et al. Analysis of CTCL cell lines reveals important differences between mycosis fungoides/Sezary syndrome vs. HTLV-1(+) leukemic cell lines. Oncotarget. 2017;8(56):95981-98.

27. Raj T, Rothamel K, Mostafavi S, Ye C, Lee MN, Replogle JM, et al. Polarization of the effects of autoimmune and neurodegenerative risk alleles in leukocytes. Science. 2014;344(6183):519-23.

28. Mifsud B, Tavares-Cadete F, Young AN, Sugar R, Schoenfelder S, Ferreira L, et al. Mapping long-range promoter contacts in human cells with highresolution capture Hi-C. Nat Genet. 2015;47(6):598-606.

29. Kumar S, Ambrosini G, Bucher P. SNP2TFBS-a database of regulatory SNPS affecting predicted transcription factor binding site affinity. Nucleic Acids Res. 2017:45(D1):D139-D44

30. Tsoi LC, Spain SL, Knight J, Ellinghaus E, Stuart PE, Capon F, et al. Identification of 15 new psoriasis susceptibility loci highlights the role of innate immunity. Nat Genet. 2012;44(12):1341-8.

31. Zhou X, Lowdon RF, Li D, Lawson HA, Madden PAF, Costello JF, et al. Exploring long-range genome interaction data using the WashU Epigenome Browser. Nat Methods. 2013;10(5):375-376.

32. Tsoi LC, Spain SL, Ellinghaus E, Stuart PE, Capon F, Knight J, et al. Enhanced meta-analysis and replication studies identify five new psoriasis susceptibility loci. Nat Commun. 2015;6:7001.

33. Maine GN, Mao X, Komarck CM, Burstein E. COMMD1 promotes the ubiquitination of NF-kappaB subunits through a cullin-containing ubiquitin ligase. EMBO J. 2007;26(2):436-47.

34. Malhotra N, Narayan K, Cho OH, Sylvia KE, Yin C, Melichar H, et al. A network of high-mobility group box transcription factors programs innate interleukin-17 production. Immunity. 2013;38(4):681-93.
35. de Cid R, Riveira-Munoz E, Zeeuwen P, Robarge J, Liao W, Dannhauser EN et al. Deletion of the late cornified envelope LCE3B and LCE3C genes as a susceptibility factor for psoriasis. Nat Genet. 2009;41(2):211-5.

36. Li M, Wu Y, Chen G, Yang Y, Zhou D, Zhang Z, et al. Deletion of the late cornified envelope genes LCE3C and LCE3B is associated with psoriasis in a Chinese population. J Invest Dermatol. 2011;131(8):1639-43.

37. Swindell WR, Sarkar MK, Liang Y, Xing X, Gudjonsson JE. Cross-disease transcriptomics: unique IL-17A signaling in psoriasis lesions and an autoimmune PBMC signature. J Invest Dermatol. 2016;136(9):1820-30.

38. Segre JA, Bauer C, Fuchs E. Klf4 is a transcription factor required for establishing the barrier function of the skin. Nat Genet. 1999;22(4):356-60.

39. An J, Golech S, Klaewsongkram J, Zhang YQ, Subedi K, Huston GE, et al. Kruppel-like factor 4 (KLF4) directly regulates proliferation in thymocyte development and IL-17 expression during Th17 differentiation. FASEB J. 2011;25(10):3634-45.

40. Hsu F, Kent WJ, Clawson H, Kuhn RM, Diekhans M, Haussler D. The UCSC known genes. Bioinformatics. 2006;22(9):1036-46.

41. Dunham I, Kundaje A, Aldred SF, Collins PJ, Davis C, Doyle F, et al. An integrated encyclopedia of DNA elements in the human genome. Nature. 2012;489(7414):57-74.

42. Kuhn RM, Haussler D, Kent WJ. The UCSC Genome Browser and associated tools. Brief Bioinform. 2013;14(2):144-61.

43. Ernst J, Kellis M. ChromHMM: automating chromatin-state discovery and characterization. Nat Methods. 2012;9(3):215-6.

44. Chen X, Whitney EM, Gao SY, Yang WW. Transcriptional profiling of Krüppellike factor 4 reveals a function in cell cycle regulation and epithelial differentiation. J Mol Biol. 2003;326(3):665-77.

45. Okano J, Opitz OG, Nakagawa H, Jenkins TD, Friedman SL, Rustgi AK. The Kruppel-like transcriptional factors Zf9 and GKLF coactivate the human keratin 4 promoter and physically interact. FEBS Lett. 2000; 473(1):95-100.

46. He H, Li S, Hong Y, Zou H, Chen H, Ding F, et al. Krüppel-like factor 4 promotes esophageal squamous cell carcinoma differentiation by upregulating keratin 13 expression. J Biol Chem. 2015;290(21):13567-77.

47. Chen X, Johns DC, Geiman DE, Marban E, Dang DT, Hamlin G, et al. Krüppel-like factor 4 (gut-enriched Krüppel-like factor) inhibits cell proliferation by blocking G1/S progression of the cell cycle. J Biol Chem. 2001;276(32):30423-8

48. Qin Q, Fan J, Zheng R, Wan C, Mei S, Wu Q, et al. Lisa: inferring transcriptional regulators through integrative modeling of public chromatin accessibility and ChIP-seq data. Genome Biol. 2020;21(1):32.

49. McGovern A, Schoenfelder S, Martin P, Massey J, Duffus K, Plant D, et al. Capture Hi-C identifies a novel causal gene, IL20RA, in the panautoimmune genetic susceptibility region 6q23. Genome Biol. 2016;17(1):212.

50. Wang SF, Wen F, Wiley GB, Kinter MT, Gaffney PM. An enhancer element harboring variants associated with systemic lupus erythematosus engages the TNFAIP3 promoter to influence A20 expression. PLoS Genet. 2013;9(9):10.

51. Simeonov DR, Gowen BG, Boontanrart M, Roth TL, Gagnon JD, Mumbach $M R$, et al. Discovery of stimulation-responsive immune enhancers with CRISPR activation. Nature. 2017;549(7670):111-5.

52. Ray SK. The transcription regulator Krüppel-like factor 4 and its dual roles of oncogene in glioblastoma and tumor suppressor in neuroblastoma. For Immunopathol Dis Therap. 2016;7(1-2):127-39.

53. Kim KJ, Park S, Park YH, Ku SH, Cho BB, Park BJ, et al. The expression and role of Kruppel-like factor 4 in psoriasis. Ann Dermatol. 2014;26(6):675-80.

54. Cheng H, Li Y, Zuo XB, Tang HY, Tang XF, Gao JP, et al. Identification of a missense variant in LNPEP that confers psoriasis risk. J Investig Dermatol. 2014;134(2):359-65.

55. Belton JM, McCord RP, Gibcus JH, Naumova N, Zhan Y, Dekker J. Hi-C: a comprehensive technique to capture the conformation of genomes. Methods. 2012;58(3):268-76.

56. Wingett $S$, Ewels $P$, Furlan-Magaril M, Nagano T, Schoenfelder $S$, Fraser $P$, et al. HiCUP: pipeline for mapping and processing Hi-C data. F1000Res. 2015;4:1310.

57. Cairns J, Freire-Pritchett P, Wingett SW, Varnai C, Dimond A, Plagnol V, et al. CHiCAGO: robust detection of DNA looping interactions in Capture Hi-C data. Genome Biol. 2016;17(1):127.

58. Picard Toolkit. Broad Institute, GitHub Repository: Broad Institute; 2019 http://broadinstitute.github.io/picard/.

59. Heinz S, Texari L, Hayes MGB, Urbanowski M, Chang MW, Givarkes N, et al. Transcription elongation can affect genome 3D structure. Cell. 2018;174(6): 1522-36.e22. 
60. Quinlan AR. BEDTools: the Swiss-army tool for genome feature analysis. Current protocols in bioinformatics/editoral board, Andreas D Baxevanis [et al]. 2014;47:11 2 1-2 34.

61. Chen S, Zhou Y, Chen Y, Gu J. fastp: an ultra-fast all-in-one FASTO preprocessor. Bioinformatics. 2018;34(17):i884-i90.

62. Servant N, Varoquaux N, Lajoie BR, Viara E, Chen CJ, Vert JP, et al. HiC-Pro: an optimized and flexible pipeline for $\mathrm{Hi}-\mathrm{C}$ data processing. Genome Biol. 2015;16:259.

63. Kruse K, Hug CB, Hernández-Rodríguez B, Vaquerizas JM. TADtool: visual parameter identification for TAD-calling algorithms. Bioinformatics. 2016; 32(20):3190-2

64. Bhattacharyya S, Chandra V, Vijayanand P, Ay F. Identification of significant chromatin contacts from HiChIP data by FitHiChIP. Nat Commun. 2019;10:14.

65. Lareau CA, Aryee MJ. hichipper: a preprocessing pipeline for calling DNA loops from HiChIP data. Nat Methods. 2018;15(3):155-6.

66. Love Ml, Huber W, Anders S. Moderated estimation of fold change and dispersion for RNA-seq data with DESeq2. Genome Biol. 2014;15(12):550.

67. Bolger AM, Lohse M, Usadel B. Trimmomatic: a flexible trimmer for Illumina sequence data. Bioinformatics. 2014;30(15):2114-20.

68. Martin M. Cutadapt removes adapter sequences from high-throughput sequencing reads. EMBnetj. 2011;17(1):2226-6089.

69. Smith T, Heger A, Sudbery I. UMI-tools: modeling sequencing errors in unique molecular identifiers to improve quantification accuracy. Genome Res. 2017;27(3):491-9.

70. Dobin A, Davis CA, Schlesinger F, Drenkow J, Zaleski C, Jha S, et al. STAR: ultrafast universal RNA-seq aligner. Bioinformatics. 2013;29(1):15-21.

71. Anders S, Pyl PT, Huber W. HTSeq--a Python framework to work with highthroughput sequencing data. Bioinformatics. 2015;31(2):166-9.

72. Luo W, Friedman MS, Shedden K, Hankenson KD, Woolf PJ. GAGE: generally applicable gene set enrichment for pathway analysis. BMC Bioinformatics. 2009;10:161.

73. Boyle AP, Hong EL, Hariharan M, Cheng Y, Schaub MA, Kasowski M, et al. Annotation of functional variation in personal genomes using RegulomeDB. Genome Res. 2012;22(9):1790-7.

74. Ward LD, Kellis M. HaploReg: a resource for exploring chromatin states, conservation, and regulatory motif alterations within sets of genetically linked variants. Nucleic Acids Res. 2012;40(Database issue):D930-4.

75. Lonsdale J, Thomas J, Salvatore M, Phillips R, Lo E, Shad S, et al. The Genotype-Tissue Expression (GTEx) project. Nat Genet. 2013;45(6):580-5.

76. Lappalainen T, Sammeth M, Friedlander MR, t Hoen PA, Monlong J, Rivas MA, et al. Transcriptome and genome sequencing uncovers functional variation in humans. Nature. 2013:501(7468):506-11.

77. Naumova N, Smith EM, Zhan Y, Dekker J. Analysis of long-range chromatin interactions using chromosome conformation capture. Methods. 2012:58(3):192-203.

78. Untergasser A, Cutcutache I, Koressaar T, Ye J, Faircloth BC, Remm M, et al. Primer3--new capabilities and interfaces. Nucleic Acids Res. 2012;40(15):e115.

79. Klann TS, Black JB, Chellappan M, Safi A, Song L, Hilton IB, et al. CRISPR-Cas9 epigenome editing enables high-throughput screening for functional regulatory elements in the human genome. Nat Biotechnol. 2017;35(6):561-8.

80. Concordet JP, Haeussler M. CRISPOR: intuitive guide selection for CRISPR/ Cas9 genome editing experiments and screens. Nucleic Acids Res. 2018; 46(W1):W242-w5.

81. Heckl D, Kowalczyk MS, Yudovich D, Belizaire R, Puram RV, McConkey ME, et al. Generation of mouse models of myeloid malignancy with combinatorial genetic lesions using CRISPR-Cas9 genome editing. Nat Biotechnol. 2014;32(9):941-6.

82. Lawhorn IE, Ferreira JP, Wang CL. Evaluation of sgRNA target sites for CRISPR-mediated repression of TP53. PLoS One. 2014;9(11):e113232.

83. Szklarczyk D, Franceschini A, Wyder S, Forslund K, Heller D, Huerta-Cepas J et al. STRING v10: protein-protein interaction networks, integrated over the tree of life. Nucleic Acids Res. 2015:43(Database issue):D447-52.

84. Perez-Pinera P, Kocak DD, Vockley CM, Adler AF, Kabadi AM, Polstein LR, et al. RNA-guided gene activation by CRISPR-Cas9-based transcription factors. Nat Methods. 2013;10(10):973-6.

85. Bernstein BE, Stamatoyannopoulos JA, Costello JF, Ren B, Milosavljevic A, Meissner A, et al. The NIH Roadmap Epigenomics Mapping Consortium. Nat Biotechnol. 2010;28(10):1045-8.

\section{Publisher's Note}

Springer Nature remains neutral with regard to jurisdictional claims in published maps and institutional affiliations.

Ready to submit your research? Choose BMC and benefit from:

- fast, convenient online submission

- thorough peer review by experienced researchers in your field

- rapid publication on acceptance

- support for research data, including large and complex data types

- gold Open Access which fosters wider collaboration and increased citations

- maximum visibility for your research: over $100 \mathrm{M}$ website views per year

At BMC, research is always in progress.

Learn more biomedcentral.com/submissions 